\title{
Trend der Schwermetall-Bioakkumulation 1990 bis 2005: Qualitätssicherung bei Probenahme, Analytik, geostatistischer Auswertung
}

\author{
Winfried Schröder · Roland Pesch · Yehia Matter · Axel Göritz $\cdot$ Lutz Genßler · Helga Dieffenbach-Fries
}

Eingegangen: 20. März 2009/Akzeptiert: 13. August 2009/ Online veröffentlicht: 25. September 2009

(C) Springer-Verlag 2009

\begin{abstract}
Zusammenfassung Hintergrund, Ziel und Bereich Aus dem Moosmonitoring liegen seit 1990 alle fünf Jahre flächendeckende Inventuren der Schwermetall-Bioakkumulation vor. Die Daten belegen flächendeckend für Deutschland und Europa, wie sich Luftreinhaltepolitik auf die Anreicherung von Metallen in Ökosystemen auswirkt, die nicht offensichtlich im Einflussbereich technischer Emissionsquellen liegen. Dieser Artikel behandelt die Kartierung der zeitlichen Entwicklung der Metallanreicherung in Deutschland in Fünfjahresschritten seit 1990 sowie die räumliche Varianz der Metall-Bioakkumulation in Abhängigkeit von Eigenschaften der Probeentnahmestellen und ihrer Umgebung. Ein besonderes Augenmerk gilt hierbei der Korrelation zwischen den Metallgehalten in Moosen und Depositionen. Damit werden Beiträge für die Umweltbeobachtung nach $\S 12$ Bundesnaturschutzgesetz und für die Genfer Luftreinhaltekonvention (Übereinkommen über den weiträumigen, grenzüberschreitenden Transport von Luftverunreinigungen, CLRTAP) geliefert.
\end{abstract}

W. Schröder $(\bowtie) \cdot R$. Pesch

Lehrstuhl für Landschaftsökologie, Hochschule Vechta,

PF 1553, 46364 Vechta, Deutschland

E-Mail: wschroeder@iuw.uni-vechta.de

Y. Matter · A. Göritz

LUFA Nord-West, Institut für Düngemittel und Saatgut,

Finkenborner Weg 1A, 31787 Hameln, Deutschland

L. Genssler

(als Obmann stellvertretend für den Arbeitskreis Bioindikation und

Wirkungsermittlung der Landesanstalten und -ämter)

Landesamt für Natur, Umwelt und Verbraucherschutz Nordrhein-

Westfalen,

Leibnizstr. 10, 45610 Recklinghausen, Deutschland

H. Dieffenbach-Fries

Umweltbundesamt - Fachgebiet II 5.4, UBA-Dienstgebäude Langen,

Paul-Ehrlich-Str. 29, 63225 Langen, Deutschland
Material und Methoden Die Anreicherung von bis zu 40 Spurenelementen in Moosen wurde nach europaweit harmonisierter Vorgehensweise bestimmt. Die hierbei angewendete Richtlinie regelt die Auswahl der Orte, an denen die Moose gesammelt werden, die zu berücksichtigenden Moosarten, ihre chemische Analyse und Qualitätskontrolle sowie die Klassierung der Messdaten für die Kartierung ihrer räumlichen Strukturen. In Deutschland wurden die bis zu 1028 Standorte des Moosmonitorings hinsichtlich topografischer und ökologischer Kriterien sowie Vorgaben der Richtlinie beschrieben. Diese Standortbeschreibungen wurden mit Informationen über Emissionen aus technischen Anlagen und Daten über die Landnutzung in der Umgebung der Moossammelstellen sowie mit den Messdaten in dem internetfähigen WebGIS MossMet zusammengeführt und ausgewertet: Die räumliche Struktur der Messdaten wurde per Variogramm-Analyse analysiert und modelliert sowie mit Kriging-Interpolation kartiert. Aus den standort- und metallspezifischen Messdaten sowie den daraus geostatistisch berechneten Flächendaten über die Metallakkumulation wurde ein zusammenfassender Multi-Metall-Index (MMI) für Cr, Cu, Fe, Ni, Pb, Ti, V und Zn für das Jahr 1990 (MMI1990) sowie jahresübergreifend (MMI1990-2005) sowie für jede Kampagne ab 1995 einzeln und zeitlich integrierend für $\mathrm{As}, \mathrm{Cd}, \mathrm{Cr}, \mathrm{Cu}, \mathrm{Fe}, \mathrm{Hg}, \mathrm{Ni}, \mathrm{Pb}, \mathrm{Sb}, \mathrm{Ti}, \mathrm{V}$ und Zn (MMI1995, MMI2000, MMI2005, MMI1995-2005) berechnet und kartiert. Die Zusammenhänge zwischen den Schwermetall-Akkumulationen, Standortcharakteristika sowie Landnutzungen und Emissionen wurden mit bivariaten Rankkorrelationskoeffizienten sowie multivariat-statistisch mit „Classification and Regression Trees“ (CART) und "Chisquare Automatic Interaction Detection“ (CHAID) quantitativ gekennzeichnet.

Ergebnisse Die Ergebnisse der qualitätskontrollierten chemischen Analysen zeigen von 1990 bis 2000 einen sta- 
tistisch signifikanten Rückgang der Metall-Bioakkumulation. Von 2000 bis 2005 ist wieder ein Anstieg der MetallBioakkumulation zu beobachten, besonders für $\mathrm{Cd}, \mathrm{Cr}, \mathrm{Cu}$, $\mathrm{Sb}$ und $\mathrm{Zn}$. Besonders deutlich sind hierbei die Zunahmen für $\mathrm{Cr}(160 \%)$, das nahezu ähnlich hohe Werte wie 1990 erreicht. Der zeitliche Trend der vergangenen 15 Jahre zeigt für die meisten Metalle jedoch einen deutlichen und flächendeckenden Rückgang der Akkumulation in Moosen. Signifikante Abnahmen seit 2000 wurden bei $\mathrm{Hg}, \mathrm{Pb}$ und Ti festgestellt. Die entsprechenden Trends für die einzelnen Bundesländer sind allerdings unterschiedlich. Die Metallgehalte in den Moosen zeigen bis auf $\mathrm{Cr}$ ähnliche räumliche Verteilungsmuster wie in den Kampagnen 1995 und 2000. Die Hotspots finden sich zumeist im urban-industriell geprägten Ruhrgebiet, der dicht besiedelten Rhein-Main-Region sowie in den industriell geprägten Regionen der neuen Länder (z. B. Raum Halle/Leipzig). Die räumliche Varianz der Metall-Bioakkumulation lässt sich u.a. durch Eigenschaften der Moossammelstellen (z.B. Moosart, Trauf-Effekt) und ihrer Umgebung (z. B. Korrelation mit Deposition, Landnutzung) erklären.

Diskussion Insbesondere der Anstieg der Cr-Bioakkumulation vom Jahr 2000 zum Jahr 2005 wurde auch in anderen Teilnehmerstaaten wie z. B. in der Schweiz bestätigt. Es bedarf weiterer Untersuchungen, ob es sich hierbei um einen emissionsbedingten oder einen biogenen Effekt (z. B. infolge gleichzeitig angestiegener Stickstoffbelastung) handelt. Im Gegensatz zu anderen Informationsquellen, wie sie z. B. die Messung und Modellierung der Deposition darstellen, hat das Moosmonitoring den Anstieg der Konzentration toxikologisch relevanter Metalle in Moosen zwischen den Jahren 2000 und 2005 registriert. Im Vergleich zu zeitlich höher aufgelösten Depositionsmessungen erfasst das Moosmonitoring großräumig ein breites Stoffspektrum, das auch in anderen Messnetzen selten gemessene Stoffe mit humantoxikologischer Bedeutung (z. B. Hg, Sb, As, Al, V) umfasst. Das standardisierte Biomonitoring von Luftverunreinigungen mit Bodenmoosen bildet folglich ein wichtiges Bindeglied zwischen der technischen Erfassung von Stoffeinträgen durch Deposition und der Anreicherung dieser Stoffe in biologischem Material. Die häufig zum Qualitätsnachweis für das Moosmonitoring erhobene Forderung, die Stoffkonzentrationen in der Deposition und in biologischem Material müsse hoch miteinander korrelieren, ist nicht sachgemäß, da es sich nicht um identische, sondern um lediglich verknüpfte Phänomene handelt. Der Betrag ihrer statistischen Korrelation hängt ab von der Ausprägung den Randbedingungen der physikalischen Prozesse, so u. a. von den regional und standörtlich im Anreicherungszeitraum vorherrschenden meteorologischen Verhältnisse, der horizontalen und vertikalen Vegetationsstruktur und der Landnutzung.

Schlussfolgerungen Das Moosmonitoring liefert wesentliche Beiträge zum Schwermetall- und zum Multi-Kompo-
nenten-Protokoll der CLRTAP, denn es belegt flächendeckend, wie sich Luftreinhaltepolitik auf die Anreicherung von Emissionen in Schutzgütern wie der Vegetation auswirkt. Wenn Umweltbeobachtung als Daueraufgabe verstanden wird und die eingesetzte Methodik für Frühwarnung geeignet ist, eröffnet sie der Umweltpolitik die Möglichkeit, vorsorgend zu handeln und überraschenden Entwicklungen nachzugehen. Von besonderer umweltpolitischer Bedeutung ist ferner, dass in keinem anderen Messprogramm räumlich so verdichtet Daten über ein breites, ökotoxikologisch relevantes Stoffspektrum erhoben werden. Die räumliche Auflösung von Umweltinformationen ist ein wesentliches Kriterium für ihre Nutzbarkeit im Vollzug umweltpolitischer Maßnahmen auf Bundes- und Länderebene.

Empfehlungen und Perspektiven Das Moosmonitoring ist ein Vorzeigeprogramm der Umweltbeobachtung, das über drei räumliche und administrative Ebenen - Region (z.B. Bundesland oder Naturraum), Staat (z. B. Deutschland) und Kontinent (Europa) - harmonisierte, qualitätskontrollierte Daten zur Umweltüberwachung und Wirksamkeitskontrolle umweltpolitischer Maßnahmen über ein internetfähiges WebGIS-Portal bereitstellt. Das Moosmonitoring erfüllt insofern eine wichtige Aufgabe im europäischen Umweltmonitoring und sollte aus umweltwissenschaftlichen und umweltpolitischen Gründen auch zukünftig in Gänze weiter fortgeführt werden.

Schlüsselwörter CART (Classification and Regression Trees) $\cdot$ CHAID (Chisquare Automatic Interaction Detection) · Geostatistik · INSPIRE (Infrastructure for Spatial Information in the European Community) . Moose - Stoffanreicherungen - Umweltkonzentration · UN/ECE CLRTAP (Übereinkommen über den weiträumigen, grenzüberschreitenden Transport von Luftverunreinigungen)

\section{Trends of metal bioaccumulation from 1990 to 2005 in Germany}

Abstract Background, aim and scope Since 1990, the UN ECE Heavy Metals in Mosses Surveys provide data inventories of the atmospheric heavy metal bioaccumulation across national boundaries in Europe. The results prove how air pollution control in Germany and in all of Europe affected the bioaccumulation of metals in those ecosystems that are not directly influenced by nearby emission sources. This article focuses on the assessment of spatiotemporal patterns of the metal bioaccumulation in Germany since 1990. Furthermore, the spatial variance of the metal bioaccumulation is analysed with regard to sampling site-specific and regional land characteristics. Special focus hereby relies on the correlation of the metal concentration in mosses and in depo- 
sitions. Hence, the moss surveys contribute to $\S 12$ of the German Federal Nature Conservation Act as well as to the "Convention on Long-range Transboundary Air Pollution" (CLRTAP).

Materials and methods The bioaccumulation of up to 40 trace elements in mosses was determined according to a European wide harmonised methodology. The according experimental protocol regulates the selection of sampling sites and moss species, the chemical analysis and quality control and the classification of the measured values for the mapping of spatial patterns. In Germany all sampling sites were described with regard to topographical and ecological criteria as well as other aspects seen as relevant in the mandatory guideline. Together with the measurements this metadata was combined with other information regarding emissions and land use in the surroundings of the sampling sites in the WebGIS MossMet. The spatial structure of the metal bioaccumulation was analysed and modeled by variogram analyses and then mapped by applying different kriging techniques. Furthermore, different multi-metal indices (MMI) were derived for both the sampling sites and raster maps with the help of percentile statistics: The MMI1990 aggregates the data for $\mathrm{Cr}, \mathrm{Cu}, \mathrm{Fe}, \mathrm{Ni}, \mathrm{Pb}, \mathrm{Ti}, \mathrm{V}$ and $\mathrm{Zn}$ determined in 1990. The MMI1995, MMI2000, MMI2005 furthermore include $\mathrm{As}, \mathrm{Cd}, \mathrm{Hg}$ and $\mathrm{Sb}$ for 1995, 2000 and 2005 , respectively. Two other MMI allow for a time integrating view on the metal bioaccumulation in Germany: The MMI1990-2005 was calculated on behalf of all measured/ geostatistically estimated data for $\mathrm{Cr}, \mathrm{Cu}, \mathrm{Fe}, \mathrm{Ni}, \mathrm{Pb}, \mathrm{Ti}, \mathrm{V}$ and $\mathrm{Zn}$. Therefore the integrated assessment of the metal bioaccumulation in Germany from 1990 to 2005 is possible. The MMI1995-2005 furthermore includes the element-specific data of $\mathrm{As}, \mathrm{Cd}, \mathrm{Hg}$ and $\mathrm{Sb}$ therefore integrating 12 elements over the last three surveys. The statistical association of the metal bioaccumulation, site-specific characteristics as well as information on land use and emissions was analysed by bivariate correlation analysis and multivariate decision tree models (Classification and Regression Trees - CART, Chisquare Automatic Interaction Detection - CHAID).

Results The results of the quality-controlled chemical analyses show a significant decrease of the metal bioaccumulation in Germany from 1990 to 2000. From 2000 to 2005 a further decrease can be stated for $\mathrm{Hg}, \mathrm{Pb}$ and $\mathrm{Ti}$. However, a significant increase for $\mathrm{Cd}, \mathrm{Cr}, \mathrm{Cu}, \mathrm{Sb}$ and $\mathrm{Zn}$ can be observed. This especially holds true for $\mathrm{Cr}(+160 \%)$ that almost reaches as high concentrations in mosses as in 1990. In 2005, the metal loads in mosses, except for $\mathrm{Cr}$, show spatial distributions similar to those in 1990, 1995 and 2000. Hot spots are mostly found in the urbanised and industrially influenced Ruhr Area, the densely populated Rhine-Main region and in the industrially influenced regions of former East Germany (e.g. Halle-Leipzig region). The spatial variance of the metal bioaccumulation can main- ly be explained by site-specific (moss species, canopy drip effects) and site-surrounding (land use, depositions, emissions) characteristics.

Discussion High Cr loads in mosses were also registered in other European countries like in Switzerland. Further investigation is therefore necessary to investigate whether this is due to different emission conditions or biogenic effects (e.g. as a result of increasing nitrogen depositions). Compared to other environmental monitoring and modelling programmes the moss surveys registered increasing concentrations of toxic metal elements between 1990 and 2005. Contrary to deposition measurements that exhibit a higher temporal resolution the moss surveys provide measurement data on a wide range of elements. Some of these elements are important with regard to human-toxicological aspects (e. g. Hg, Sb, As, Al, V). The standardised biomonitoring of atmospheric pollution by mosses is an important link between the technical acquisition of depositions and the accumulation in biological material. To claim that the element concentrations in mosses and in the deposition should correlate to a high degree is not appropriate since both approaches are physically related but are not identical. The degree of correlation thereby depends on the boundary conditions of the physical processes, like regional and sitespecific meteorological conditions within the accumulation period, the vertical and horizontal vegetation structure or land use conditions.

Conclusions The moss surveys contribute to the heavy metal and multi-component model of CLRTAP because they prove on different spatial scales how air pollution control influences the accumulation of emitted substances in environmental subjects of protection like vegetation. If environmental monitoring is seen as a continuous task and the applied methodology works well as an early warning system then environmental policy is enabled to act in preventative sense and to pursue unexpected developments. No other environmental monitoring programme provides such a wide range of ecotoxically relevant elements measured as spatially dense as the case for the moss surveys. The spatial distribution of environmental information is an essential criterion for their usability in terms of political measures for the federal states and the federation.

Recommendations and perspectives The Heavy Metals in Mosses Surveys are a positive example for environmental monitoring activities reaching across three spatial and administrative levels: regional (e.g. federal state or natural landscape), nationwide (e. g. Germany) and continental (e. g. Europe). In Germany the harmonised and quality-controlled moss data are made available via an internet-based webGIS portal. Therefore the moss data may easily be accessed for environmental monitoring purposes and the control of environmental political actions. Hence, the monitoring of Heavy Metals by Mosses Surveys is an important task among the 
European environmental observations, which should be continued in future for scientific and political reasons in its current extent.

Keywords Accumulation of substances - CART (Classification and Regression Trees) · CHAID (Chisquare Automatic Interaction Detection) - Geostatistics · INSPIRE (Infrastructure for Spatial Information in the European Community) $\cdot$ Mosses $\cdot$ Heavy metals $\cdot$ Predicted environmental concentration · UN/ECE CLRTAP (Convention on Long-range Transboundary Air Pollution)

\section{Hintergrund, Ziel und Bereich}

Unter der Schirmherrschaft der Europäischen Wirtschaftskommission (UNECE) arbeiten 51 Staaten im Rahmen der Genfer Luftreinhaltekonvention (Übereinkommen über den weiträumigen, grenzüberschreitenden Transport von Luftverunreinigungen, CLRTAP) an der Verminderung der grenzüberschreitenden Luftverschmutzung in Europa. In diesem Rahmen wird im Fünfjahresturnus an mehr als 6000 Probenentnahmestandorten in bis zu 28 europäischen Staaten das Moosmonitoring durchgeführt, um die Konzentrationen von Schwermetallen (seit 1990) und Stickstoff (seit 2005) mit hoher räumlicher Auflösung zu ermitteln. In keinem anderen Umweltmessprogramm wird eine so breite Palette ökotoxikologisch relevanter Stoffe räumlich so verdichtet erhoben; in anderen Programmen erfolgt oft nur eine räumlich stark eingeschränkte Messung weniger Elemente.

Die Daten des Moosmonitorings sind ein wissenschaftlicher Beitrag zum Schwermetall- und zum Multi-Komponenten-Protokoll der CLRTAP. Eine europaweite Auswertung und Darstellung der Ergebnisse erfolgt in Berichten der Working Group on Effects (WGE) der CLRTAP und des Programmzentrums des International Cooperative Programme on Effects of Air Pollution on Natural Vegetation and Crops (ICP Vegetation) in Bangor, Wales (http://icpvegetation.ceh.ac.uk/).

Deutschland hat sich an allen bisherigen Messkampagnen beteiligt. Über die Entwicklung der Metallakkumulation in Moosen bis zum Jahr 2000 berichten Schröder und Pesch $(2004,2005)$.

Ziel dieses Beitrags ist es, diese Berichterstattung fortzusetzen und wesentliche methodische Bestandteile der Auswertungsstrategie vorzustellen, die zukünftig Leitlinie für die Auswertung der Daten aller Teilnehmerstaaten sein soll. Ziel dieser Auswertungen ist es, die räumliche Variabilität der Messdaten durch ihre Verknüpfung mit Merkmalen, welche die Moossammelorte und ihre Umgebung kennzeichnen, zu beschreiben. Die dabei erzielten Ergebnisse sollen ferner dafür genutzt werden, die räumlichen Unschärfen der Depositionsmodellierung zu mindern.
Grundlage der Bewertung von Stoffen in der Umwelt ist der Vergleich ihrer Exposition (Konzentration in der Umwelt) und ihrer Wirkung in für sie relevanten Umweltkompartimenten. Die Konzentration, mit der ein Stoff in der Umwelt vorkommt (Predicted Environmental Concentration - PEC) wird in Beziehung zu derjenigen Konzentration gesetzt, bei der voraussichtlich noch keine Wirkungen auf Organismen oder Ökosysteme auftreten (Predicted NoEffect-Concentration - PNEC). Die Umweltkonzentration kann anhand der aufeinander folgenden Vorgänge Emission (EM), Immission (IM), Deposition (DEP) oder Akkumulation (AKK) von Stoffen bestimmt werden. Da die Messwerte oft nur für relativ wenige Orte vorliegen, wird die Umweltkonzentration von Stoffen vielfach geschätzt. Entsprechend der genutzten Datengrundlage können PECEM, PECIM, PECDEP oder PECAKK unterschieden werden, deren Informationsgehalte einander ergänzen. Wenn PECWerte größer sind als die PNEC, liegt ein Risiko vor und die zuständige Behörde entscheidet über das weitere Vorgehen (Fränzle et al. 1995). Das Moosmonitoring liefert PECAKK-Werte. Da die Deposition und Akkumulation von Stoffen unmittelbar miteinander verknüpfte Vorgänge sind, werden die aus dem Moosmonitoring hervorgehenden Daten vielfach als Surrogate für räumlich weit weniger differenziert vorliegende Daten über Stoffeinträge durch Deposition verwendet. Dies ist dann sinnvoll, wenn zwischen den Konzentrationen von Stoffen in Depositionen und Moosen hinreichend enge und signifikante statistische Beziehungen nachgewiesen werden.

Da Moose über mehrere Jahre die Anreicherung von Metallen ohne physiologische Beeinträchtigung tolerieren, sind sie besonders geeignet für das Monitoring der Bioakkumulation atmosphärischer Depositionen (Forster et al. 1993; Genßler et al. 2001; Herpin et al. 2004; Kostka-Rick et al. 2001; Mohr 1999, 2007; Rühling und Tyler 1968, 1969, 1970; Schröder et al. 2008; Tyler 1990; Wappelhorst et al. 2000; Zechmeister et al. 2006). Für großräumige Untersuchungen räumlicher Trends gilt dies im besonderen Maße (Bealey 2008 a,b). Deswegen wird das Moosmonitoring in Europa seit 1990 alle fünf Jahre in mindestens 21 Ländern durchgeführt (Harmens et al. 2008). Ziel ist es, die großräumigen Muster und zeitlichen Entwicklungen der Metallbioakkumulation an Orten ohne direkt benachbarte Emissionsquellen staatenübergreifend zu kartieren.

\section{Material und Methoden}

\subsection{Moosproben-Sammlung}

Deutschland beteiligte sich am Moosmonitoring 1990, 1995, 2000 and 2005, wobei die jeweils aktuell europaweit gültige Richtlinie angewendet wurde (Harmens 2005). 
Die räumliche Dichte des Moosmessnetzes übertraf mit 1,7 (1990), 2,9 (1995, 2000) und 2,0 (2005) Standorten pro $1000 \mathrm{~km}^{2}$ den Mindestwert von 1,5 Moosproben pro $1000 \mathrm{~km}^{2}$. Die Messnetzausdünnung beim Monitoring in 2005 erfolgte ohne Einbußen der geostatistischen Validität und Landschaftsrepräsentanz des Messnetzes (Pesch et al. 2008, Schröder et al. 1991, 2004; Schröder und Schmidt 2000). Das internetfähige geografische Informationssystem WebGIS MossMet ermöglichte die interaktive webgestützte Dokumentation von standortbeschreibenden Metadaten seitens der Probenentnehmer (Kleppin et al. 2008b; Pesch et al. 2007a). Die Anwendung erfüllt die für PortalU (http:// www.portalu.de/) sowie die „Infrastructure for Spatial Information in the European Community" (INSPIRE, http:// inspire.jrc.ec.europa.eu/) maßgeblichen technischen Standards und macht Ergebnisse der vier MoosmonitoringKampagnen zugänglich:

1. standortspezifische Informationen (s.u.) im Sinne der Verfahrensrichtlinie (ICP Vegetation 2005), ökologische und topografische Standorteigenschaften, Ablauf und meteorologische Randbedingungen der Moosprobenentnahme,

2. standortspezifische Messdaten von bis zu 40 Spurenelementen der Monitoring-Kampagnen 1990, 1995, 2000, und 2005,

3. flächenhafte Informationen über das Klima, die Höhenlage über NN, Landnutzung, Entfernung zu großen Industriebetrieben und deren Emissionen gemäß europäischem Schadstoffemissionsregister EPER sowie Depositionen an Messstellen in der Umgebung der MoosmonitoringStandorte.

Die Moosprobenentnahme erfolgte in Anlehnung an die maßgeblichen Richtlinie (ICP Vegetation 2005). Demnach gilt (Harmens 2005):

1. Die Sammelorte sollen ländliche Gegenden repräsentieren und mindestens $300 \mathrm{~m}$ von Fernverkehrsstraßen, Ortschaften und mindestens $100 \mathrm{~m}$ von kleinen Straßen und Häusern entfernt sein.

2. Die Moose sollen auf dem Boden oder auf Baumstümpfen mindestens $3 \mathrm{~m}$ entfernt von Bäumen gesammelt werden, vorzugsweise in kleinen Lichtungen innerhalb von Wäldern, also ohne Traufeinflüsse.

3. Die Sammlung von Moosen unter Sträuchern oder großblättrigen Pflanzen der Krautschicht ist ebenso zu vermeiden wie die Sammlung von Moosen auf Abhängen mit oberflächlichem Abfluss.

4. In Gebirgen sollen die Moose unterhalb der Baumgrenze entnommen werden.

5. Um zeitliche Trends zuverlässig identifizieren zu können, sollen die Orte der Moosprobensammlung mit denen der jeweils vorangegangenen Kampagne übereinstimmen, d.h. nicht mehr als zwei Kilometer von ihnen entfernt sein.
6. Sammelorte sollen möglichst nahe an langfristig betriebenen Depositionsmessstellen gelegen sein, um die Metallkonzentrationen den atmosphärischen Depositionen räumlich zuordnen zu können und auf dieser Grundlage die Korrelation untersuchen zu können.

7. Für jeden Sammelort soll eine Mischprobe aus fünf bis zehn Teilproben, entnommen aus einer $50 \times 50 \mathrm{~m}^{2}$ umfassenden Fläche, gebildet werden. Die Mischprobe soll nur Moos einer Art enthalten und ein Volumen von rund einem Liter umfassen.

8. Die Teilproben sollen nebeneinander oder übereinander in Papier- oder Plastik-Tüten angemessenen Volumens verpackt werden.

9. Das Rauchen ist während der Probenentnahme und während der gesamten Probenbehandlung untersagt. Bei der Einsammlung der Moose sind die Hände mit Kunststoffhandschuhen oder -tüten zu überziehen. Mit Talkum behandelte Laborhandschuhe dürfen nicht verwendet werden.

10. Die Moosproben sollen vorzugsweise zwischen April und Oktober in kürzestmöglicher Zeit gesammelt werden.

11. Jeder Sammelort muss durch geografische Koordinaten, vorzugsweise Länge und Breite (Greenwich, 360'-System), gekennzeichnet werden.

\subsection{Chemische Analytik und Qualitätssicherung}

Die Qualitätskontrolle wurde zweistufig ausgestaltet: Sie erfolgte zunächst in den einzelnen Teilnehmerstaaten und umfasste im Wesentlichen: 1. die Messung der Referenzmaterialien M2 (hohe Metallkonzentrationen) und M3 (Hintergrundwerte) in jeder Messserie sowie 2. den Austausch und die Messung von Moosproben, die auf beiden Seiten der Grenzen benachbarter Staaten gesammelt wurden. Anschließend wurden alle qualitätskontrollierten Daten der Teilnehmerstaaten vom Programmzentrum des ICP Vegetation erneut daraufhin überprüft, ob sie den anhand der Referenzstandards M2 und M3 bestimmten Anforderungen nach Steinnes et al. (1997) entsprechen. Smodiš and Bleise (2007) ergänzten die vom Programmzentrum durchgeführte Überprüfung der Qualitätskontrollkenndaten durch eine eigenständige Qualitätskontrollstudie, in der die Referenzmaterialien M2 und M3 von 17 Laboratorien aus 15 Staaten untersucht wurden. Die wesentlichen Befunde werden in der Diskussion mit den Ergebnissen der Qualitätssicherung im deutschen Moosmonitoring verglichen (Abschn. 4.2).

In den deutschen Moosmonitoring-Kampagnen wurde mit großer Sorgfalt vorgegangen - beginnend mit dem Messnetzdesign über die Schulung der Moosprobensammler, die Probenentnahme einschließlich Dokumentation, die chemische Analytik und Qualitätskontrolle bis zur Datenauswertung (Schröder et al. 1991, 2004). Zur Sicherung und 
Kontrolle der analytischen Qualität wurden alle üblichen Maßnahmen ergriffen (Funk et al. 2006), bezogen auf das Moosmonitoring bedeutete dies ein mehrstufiges Verfahren: Teilnahme an internationalen Ringuntersuchungen, Untersuchung von Proben aus vorangegangenen Moosmonitoring-Kampagnen, Untersuchung von Moos-Referenzproben M2 und M3, Mehrfachuntersuchung der Proben aus der Kampagne 2005, Doppelbestimmung von 120 der 720 Moosproben, Nachuntersuchung von $10 \%$ der restlichen 600 von 720 Proben, Austausch und Messung von Proben beiderseits der Grenzen unmittelbarer Nachbarn Deutschlandlands, Kontrolle auffälliger Messwerte (hier: $\mathrm{Cr}$ ) durch ein weiteres Labor (Landesamt für Natur, Umwelt und Verbraucherschutz Nordrhein-Westfalen - LANUV).

Die von den Probenentnehmern der Bundesländer an die für die chemische Analytik zuständige LUFA Nord-West verschickten Moosproben wurden beim Eintreffen im Labor zunächst mit einer Untersuchungsnummer versehen, fotografiert und, sofern sie nicht direkt weiterbearbeitet wurden, bei $8^{\circ} \mathrm{C}$ im Dunkeln zwischengelagert. Nach der Bestimmung der Moosart erfolgte die Präparation der Moosproben, wobei eine gleichbleibend hohe Qualität sichergestellt wurde. Die Proben wurden entsprechend der europäischen Richtlinie (Harmens 2005) von anhaftendem Fremdmaterial (Blätter, Wurzeln, Humuspartikel usw.) gesäubert, entsprechend der Empfehlungen von Siewers et al. (2000) jedoch keiner weiteren Waschprozedur unterzogen. Die Abtrennung der grünen, einen Akkumulationszeitraum von ca. drei Jahren repräsentierenden Moosspitzen erfolgte z.T. mithilfe einer Kunststoffschere, um so $20-40 \mathrm{~g}$ je Probe in einer Kunststoffschale zu sammeln. Von jeder der derart präparierten Proben wurden Fotografien angefertigt.

Die Trocknung der präparierten Moosproben erfolgte mind. 10 Stunden bei $70^{\circ} \mathrm{C}$ im Trockenschrank. Nach der Trocknung wurden eventuell am Schalenboden angesammelter Staub verworfen und die Moosproben gemahlen. Der Aufschluss der derart vorbehandelten Moosproben erfolgte mittels mikrowellenbeheizten Druckaufschlusses (VDLUFA-Methode): Unter Verwendung von 80-mlPTFE-TFM-Probengefäßen wurden 0,75 bis $0,85 \mathrm{~g}$ des fein gemahlenen Mooses auf $1 \mathrm{mg}$ genau eingewogen. Nach $\mathrm{Zu}-$

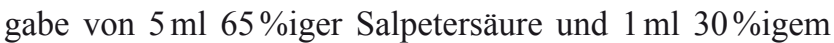
Wasserstoffperoxid (beide zur Spurenanalyse geeignet) wurden die Gefäße verschlossen und in das Aufschlusssystem (Mikrowelle MLS 1200 Mega und MLS-Ethos plus) eingesetzt. Vor dem Druckaufschluss erfolgte eine Entlüftung der Gefäße, und parallel zu jeder Serie wurde eine Blindwertprobe aufgeschlossen. Der Druckaufschluss wurde mit 250 Watt gestartet und stetig bis auf 600 Watt erhöht. Das Aufschluss-Programm endete jeweils nach 19 Minuten. Nach dem Abkühlen wurden die Probengefäße aus dem Gerät entnommen, geöffnet und ca. 20 Minuten unter dem Abzug stehengelassen. Anschließend wurde die Aufschlusslö- sung in einem 25-ml-Messkolben mit destilliertem Wasser überspült und unfiltriert analysiert.

Die Massenkonzentrationen der Elemente $\mathrm{Al}, \mathrm{Ba}, \mathrm{Ca}$, $\mathrm{Cu}, \mathrm{Fe}, \mathrm{K}, \mathrm{Mg}, \mathrm{Mn}, \mathrm{Na}, \mathrm{Sr}$, Ti und $\mathrm{Zn}$ wurden in der Aufschlusslösung mit dem ICP-OES Optima 300 der Firma Perkin-Elmer (Ecelle-Polychromator Optik, Segmentierter „Charge coupled device“-Detektor, Wellenlängenbereich UV und VIS) nach DIN EN ISO 11885 (E 22) und VDLUFA Methodenbuch 2.2.2.6 gemessen. Die Messung der Massenkonzentrationen der Elemente $\mathrm{As}, \mathrm{Cd}, \mathrm{Co}, \mathrm{Cr}, \mathrm{Mo}, \mathrm{Ni}, \mathrm{Pb}$, $\mathrm{Sb}$ und V erfolgte mit dem ICP-MS System 7500c der Firma Agilent (Shield Thorch, Octapol Massendetektor, Gaskollisionszelle) nach DIN 38406-29 (E 29) und VDLUFA Methodenbuch 2.2.2.5. Die Bestimmung der Hg-Gehalte in den Moosen erfolgte mit dem PS200 Quecksilberanalysator der Firma Leeman Labs (Thermostatisierte Zweiwegzelle) nach DIN EN 1483 und VDLUFA Methodenbuch VII.

Die Massenkonzentration vom Gesamt-N wurde mit einem C/N 2000 Analyser der Firma Leco (Wärmeleitfähigkeitsdetektor) nach VDLUFA Methodenbuch II 3.5.2.7 durch die Verbrennung von 0,2 g Moosprobenmaterial im Sauerstoffstrom bestimmt. Bei dieser Methode wird Stickstoffoxid zu molekularem $\mathrm{N}$ reduziert und dieser mittels eines Wärmeleitfähigkeitsdetektors detektiert.

Seit über 20 Jahren nimmt die LUFA Nord-West an einem internationalen Ringversuch der Universität Wageningen teil. Dabei werden in vierteljährlichen Abständen die Gehalte von über 20 Elementen in verschiedenen Pflanzenproben ermittelt. Die Auswertung erfolgt dabei nach dem statistischen Medianwertkonzept (van Dijk and Houba 1999). Im Hinblick auf die Vergleichbarkeit der in den Moosen bestimmten Elementgehalte in den Kampagnen 1995 und 2000 erfolgte in 2000 eine Analyse von Rückstellproben aus dem Moosmonitoring 1995 (Schröder et al. 2002; MARSEP 2000).

Die Überprüfung der Zuverlässigkeit der Ergebnisse der chemischen Analysen erfolgte anhand der oben erwähnten Moos-Referenzmaterialien (M2 und M3), die im internationalen Moosmonitoring 2005 europaweit einzusetzen waren. Die zwei Referenzmaterialien wurden 1993 in Finnland gesammelt, an 76 Labore aus 19 europäischen Ländern verteilt und dort mithilfe unterschiedlicher instrumenteller Analysemethoden quantitativ untersucht (Steinnes et al. 1997). Die analysierten Ergebnisse wurden anschließend zusammengefasst und statistisch aufbereitet. Für 44 Elemente wurden Referenzwerte abgeleitet. Als recommended wurden Referenzwerte bezeichnet, die in guter Übereinstimmung mit mindestens zwei der eingesetzten Analysemethoden lagen, ansonsten wurden die ermittelten Referenzwerte als indicated bezeichnet (Steinnes et al. 1997). Zum Abgleich der von Steinnes et al. (1997) ermittelten Referenzwerte mit den im Labor der LUFA Nord-West gemessenen wurden die zwei Referenzproben M2 und M3 in einem ersten Schritt jeweils viermal aufgeschlossen und auf die infrage kom- 
menden Elemente analysiert. Aus den daraus resultierenden Ergebnissen wurden Mittelwerte und Standardabweichungen berechnet und den vorgegebenen Referenzwerten und Standardabweichungen gegenübergestellt.

Bei jeder Messserie wurden jeweils eine M2- und eine M3-Referenzprobe mit in die Analysen einbezogen und die daraus resultierenden Messwerte in eine Kontrollkarte eingetragen. Es wurde festgelegt, dass die Analysenwerte für M2 und M3 bestenfalls nicht mehr als $+/-10 \%$, jedoch höchstens nicht mehr als $+/-2$ Standardabweichungen von vorgegebenen Referenzwerten abweichen sollten. War dies doch der Fall, wurde die Serie überprüft, das Messsystem optimiert und die Messung wiederholt.

Doppelbestimmungen erfolgten für die ersten 120 Moosproben. Bei Abweichungen von mehr als 10\% wurde die Probe einer dritten Untersuchung unterzogen. Danach wurde ein Mittelwert gebildet. Die Messung der restlichen 600 Moosproben erfolgte einfach, wobei im Nachhinein 10\% der Proben mit sehr niedrigen und sehr hohen Gehalten nochmals analysiert wurden. Die Werte der ersten Messung galten als bestätigt, wenn die Abweichungen zu diesen Analyseergebnissen weniger als $10 \%$ betrugen. Schließlich erfolgten noch grenzüberschreitende Qualitätsprüfungen: Mit den an Deutschland angrenzenden Teilnehmerstaaten Belgien, Frankreich, Österreich, Polen, Schweiz und Tschechien wurden jeweils fünf Moosproben ausgetauscht und analysiert.

\subsection{Plausibilitätsprüfung}

Die Plausibilitätsprüfung der Daten wurde wie die Qualitätskontrolle zweistufig ausgeführt: Bevor die qualitätskontrollierten Daten von den Teilnehmerstaaten an das Programmzentrum zur integrativen Plausibilitätsprüfung aller europaweit gemessenen Daten (Abschn. 4.3) übermittelt wurden, erfolgte eine Plausibilitätsprüfung in jedem Teilnehmerstaat. Hierzu wurde in Deutschland den Bundesländern eine Zusammenstellung deskriptiv-statistischer Kenngrößen der Analysendaten übergeben. Die tabellarische Auflistung enthielt die für jedes Bundesland gemessenen Stoffkonzentrationen einschließlich der Extremwerte der zwölf Standardelemente ${ }^{1}$ und N sowie der Elemente Al, Ba, $\mathrm{Ca}, \mathrm{Co}, \mathrm{K}, \mathrm{Na}, \mathrm{Mg}, \mathrm{Mn}, \mathrm{Mo}, \mathrm{S}$ und $\mathrm{Sr}$. Als extrem wurden solche Messwerte gekennzeichnet, die entweder über dem bundes- oder dem landesweiten 98. Perzentil lagen. In Ergänzung dazu bot das WebGIS MossMet die Möglichkeit, die standortbezogenen Mess- und Metadaten zusammen mit anderen Geoinformationen u. a. zur Landnutzung in der Umgebung der Moosproben-Entnahmeorte zu recherchie-

\footnotetext{
${ }^{1}$ Als Standardelemente werden im deutschen Moosmonitoring die Elemente $\mathrm{As}, \mathrm{Cd}, \mathrm{Cr}, \mathrm{Cu}, \mathrm{Fe}, \mathrm{Hg}, \mathrm{Ni}, \mathrm{Pb}, \mathrm{Sb}, \mathrm{Ti}, \mathrm{V}$ und $\mathrm{Zn}$ bezeichnet.
}

ren und kartografisch zu veranschaulichen. Die über dem 98. Perzentil liegenden Messwerte und andere auffällige Ergebnisse wurden pro Element und Messpunk den Ergebnissen der Messkampagnen vor 2005 gegenübergestellt und ermittelt, welche Rangstufe die im Monitoring 2005 auffälligen Messpunkte bei den vorangegangenen Kampagnen eingenommen hatten und wie hoch die prozentualen $\mathrm{Ab}$ weichungen ausfielen. Anhand dieser Informationen wurde dann eingeschätzt, ob dieser Wert der regional zu erwartenden Belastung entspricht. Traf dies zu, dann wurde der Wert mit dem Vermerk plausibel versehen, wenn nicht, dann wurde der Wert zur Überprüfung vorgesehen. Die Überprüfung erfolgte sodann durch Identifikation möglicher Einflussfaktoren, die zu erhöhten Werten und starken Unterschieden zwischen den Messkampagnen geführt haben könnten. Hierzu wurden die über das WebGIS MossMet zugänglichen Probenentnahmeprotokolle und Standortbeschreibungen analysiert. Auch die Befragung der Probensammler und ortskundiger Fachleute zur Emissionssituation gehörten zur Klärung der Plausibilität. Erschien der gefundene Wert auch danach zu hoch und nicht durch offensichtliche Einflussfaktoren erklärbar, so wurde entschieden, den Wert für nicht plausibel zu erklären und ihn aus der Auswertung herauszunehmen. (Abschn. 3.3).

\subsection{Geostatistik}

Geostatistische Verfahren wurden eingesetzt, um die räumlichen Muster der Bioakkumulation einzelner Metallelemente oder in Form des Metalle übergreifenden Multi-Metall-Index (MMI, s. u.) zu analysieren, zu modellieren und zu kartieren. Die Geostatistik basiert auf der Annahme, dass Messwerte, die an nahe beieinander liegenden Orten erhoben wurden, eine geringere Varianz aufweisen als ferner voneinander liegende. Ob dies zutrifft, wird mit der Variogramm-Analyse geprüft. Lässt sich die angenommene räumliche Autokorrelation ermitteln und durch eine Modellfunktion hinreichend beschreiben, können standortspezifische Messwerte anhand der Funktion mit Kriging-Verfahren interpoliert und so flächenhafte Schätzwerte berechnet werden (Matheron 1965; Webster und Oliver 2001).

Wenn die Varianzen mit zunehmender Messpunktdistanz steigen, so weist dies auf eine distanzabhängige Struktur der Messdaten hin. Dieser Zusammenhang ist durch ein geeignetes, z. B. sphärisches oder exponentielles Modellvariogramm anzupassen. Die maximale räumliche Aussagekraft der Messwerte ergibt sich aus der Projektion des Scheitelpunkts der Modellvariogrammkurve auf die Distanzachse. Innerhalb dieses Range (= Reichweite) wird eine Interpolation zwischen Messpunkten als statistisch sinnvoll angesehen. Die dem Range zugeordnete Semivarianz wird als Sill bezeichnet. Bei starken Messwertvariabilitäten benachbarter Messpunkte sowie bei Vorhandensein 
von Messfehlern kann es sein, dass das Modellvariogramm die Ordinate oberhalb des Ursprungs des Koordinatensystems schneidet. Der Abstand zwischen Schnittpunkt und Ursprung wird dann als Nugget-Effekt bezeichnet. Beträgt das Verhältnis von Nugget-Effekt zum Sill 1, liegt ein reiner Nugget-Effekt und somit keine räumliche Autokorrelation vor.

Die Qualität der Flächenschätzung wurde per Kreuzvalidierung ermittelt (Johnston et al. 2001; Pesch 2003; Pesch et al. 2007b). Bei der Kreuzvalidierung wird nacheinander je ein Wert aus der Gesamtmenge der Messwerte entnommen und durch Kriging mit dem gewählten Modellvariogramm neu geschätzt. Die Differenzen zwischen den Messund Schätzwerten können anschließend durch geeignete statistische Kennzahlen beschrieben werden: So verweist beispielsweise der Mittlere Fehler (Mean Error ME) auf Über- oder Unterschätzungstendenzen des Modellvariogramms. Der ME berechnet sich aus den durchschnittlichen Abweichungen zwischen Mess- und Schätzwerten und ist im Idealfall 0. Will man mehrere Messgrößen mit unterschiedlichen Skalenausdehnungen miteinander vergleichen, bietet sich die Berechnung des Medians der prozentualen Differenzen zwischen Mess- und Schätzwerten an (Median of Percental Errors - MPE) (Pesch et al. 2007b).

\subsection{Zeitliche Entwicklung}

der elementspezifischen Bioakkumulation

und der Multi-Metal Indices (MMI)

Die Multi-Metall-Indizes wurden auf Grundlage der Perzentilstatistik der Messdaten zur Metall-Bioakkumulation berechnet. Perzentile werden in der (Öko-)Toxikologie zur Beschreibung von Messwertverteilungen im zeitlichen Verlauf häufig verwendet (Sparks 2000). Ein Perzentil ist derjenige Wert einer Messgröße, der von einem bestimmten Prozentanteil der Messwerte unterschritten wird. Das 20. Perzentil ist also derjenige Messwert in einer von niedrigen zu hohen Werten geordneten Messwertreihe, der von $20 \%$ der Messwerte unterschritten wird. Der MMI wurde sowohl für die Messwerte als auch für die aus ihnen berechneten flächenhaften Schätzwerte ermittelt. Dazu wurden vorerst pro Element 10 Perzentilklassen gebildet. Jedem Perzentil wurde dann ein Indexwert zwischen 1 (niedrige Bioakkumulation) und 10 (hohe Bioakkumulation) zugewiesen. Der MMI1990-2005 eines jeden Moossammelortes oder einer jeden Rasterzelle der geostatistisch berechneten Flächenkarten entspricht dem Durchschnitt der dort berechneten elementspezifischen Indexwerte der Konzentrationen von $\mathrm{Cr}, \mathrm{Cu}, \mathrm{Fe}, \mathrm{Ni}, \mathrm{Pb}, \mathrm{V}$, Ti und $\mathrm{Zn}$, die durchgängig 1990, 1995, 2000 und 2005 in Deutschland gemessen wurden. Der MMI1995-2005 berücksichtigt zusätzlich As, Cd, Hg und Sb. Ferner zeigen die MMI1990, MMI1995, MMI2000 und MMI2005 die durchschnittli- chen elementspezifischen Indexwerte bezogen auf jeweils eine Kampagne.

2.6 Zusammenhänge der Bioakkumulation mit Deposition sowie Eigenschaften der Moossammelorte und ihrer Umgebung

Die elementspezifischen Messdaten von bis zu 40 Spurenelementen der Monitoring-Kampagnen 1990, 1995, 2000 und 2005 sowie die daraus berechneten MMI wurden im GIS mit folgenden Informationen verknüpft: 1. standortspezifische Informationen (s. u.) im Sinne der Verfahrensrichtlinie (ICP Vegetation 2005), ökologische und topografische Standorteigenschaften, Ablauf und meteorologische Randbedingungen der Moosprobenentnahme, 2. flächenhafte Informationen über das Klima, die Höhenlage über NN, Flächenanteile agrarischer oder urbaner Landnutzung nach CORINE Land Cover (Keil et al. 2005), Entfernung zu großen Industriebetrieben und zu Straßen in der Umgebung der Moosmonitoring-Standorte. Hierbei handelt es sich um Daten über atmosphärische Depositionen aus dem EMEP-Luftmessnetz des Umweltbundesamtes (wet only), aus dem ICP-Forests Level II-Messnetz und dem ICP Integrated Monitoring (Freiland- und Bestandesdeposition) sowie Flächenschätzungen der modellierten Nass-, Trocken- und Gesamtdeposition. In den Korrelationsanalysen (Abschn. 2.6.1) wurden diese Depositionsdaten mit den Stoffanreicherungen in Moosen derjenigen Moossammelorte verknüpft, die nicht weiter als $2 \mathrm{~km}$ von den Depositionsmessstationen entfernt sind.

\subsubsection{Bivariate Korrelationsanalyse}

Stärke und Richtung der statistischen Zusammenhänge zwischen a) jeweils zwei Metallgehalten in den Moosen, b) zwischen Metallkonzentrationen in Moosen und Depositionen (bulk und wet only Freiland, bulk Kronentraufe) und c) zwischen Metallgehalten und modellierter Nass-, Trockenund Gesamtdeposition wurden korrelationsstatistisch quantifiziert. Die Wahl des Zusammenhangsmaßes wurde von der Skalendignität der Daten i. S. v. Stevens (1946) sowie ihren Verteilungscharakteristika abhängig gemacht. Aufgrund nicht durchgängig normalverteilter Messwerte wurde der Rankkorrelationskoeffizient nach Spearman $r s$ berechnet. Dieser misst die Signifikanz, Stärke und Richtung der Korrelationen von Rangplätzen, welche jeweils zwei Messwerte in zwei miteinander verglichenen Messwertreihen einnehmen. Wie der Produkt-Moment-Korrelationskoeffizient rp nach Pearson, der Normalverteilung voraussetzt, rangiert $r s$ zwischen +1 und -1 . Innerhalb dieses Intervalls $[-1,+1]$ wird die die Stärke der Korrelation wie folgt bewertend klassiert (Hagl 2008): $r s$-Werte $<|0.2|$ sind sehr niedrig, zwischen $|0.2|$ und $|0.5|$ niedrig, von $|0.5|$ bis $|0.7|$ moderat, zwischen $|0.7|$ und $|0.9|$ hoch und $>|0.9|$ sehr hoch. 


\subsubsection{Multiple Korrelationsanalyse mit Entscheidungsbaumverfahren CART und CHAID}

Neben bivariaten Korrelationsanalysen wurden auch multivariate Decision Tree Models eingesetzt. Solche Entscheidungsbaumverfahren dienen der Identifikation von Strukturen in Datensätzen, in denen eine Vielzahl an Objekten anhand zahlreicher Ausprägungen kategorialer und metrischer Merkmale beschrieben wird. Ziel ist es, anhand der statistischen Beziehung zwischen einer interessierenden Variable (Zielvariable) und mehreren mit dieser in einem fachlichen Zusammenhang stehenden beschreibenden Variablen (Prädiktoren) ein Erklärungs- oder Vorhersagemodell für die interessierende Variable abzuleiten. Es existieren unterschiedliche Entscheidungsbaumalgorithmen, von denen zwei hier angewendet wurden: Classification and Regression Trees (CART, Breimann et al. 1984) sowie Chi-square Automatic Interaction Detection (CHAID, Kass 1980).

Entscheidungsbaumverfahren segmentieren einen Datensatz in einer Folge binärer (CART) oder multipler Teilungen (CHAID). Dabei wird der Ausgangsdatensatz schrittweise in Gruppen (Klassen, Knoten) zerlegt, was in Form von Dendrogrammen nachvollzogen werden kann. Die Teilungen erfolgen anhand der Merkmalsausprägungen der zur Verfügung stehenden Prädiktorvariablen. Dabei wird derjenige Prädiktor zur Teilung herangezogen, welcher im Vergleich zu allen anderen Prädiktoren ein statistisches Zielkriterium optimiert. Dabei gehen das CART- und das CHAID-Verfahren unterschiedlich vor: CART wählt aus allen beschreibenden Variablen denjenigen Prädiktor als Teilungs- oder Splitvariable aus, der den größten Zuwachs an Homogenität (Improvement) in Bezug auf die Ausprägung der Zielvariable erreicht. Die Bestimmung der gruppeninternen Homogenität basiert bei metrisch skalierten Zielvariablen auf der klasseninternen Varianz und das Improvement pro Split auf der Verringerung der Varianz des Ausgangsdatensatzes in Bezug auf die Varianz der durch die Teilung gebildeten neuen Baumebene. CHAID bemisst den Zusammenhang zwischen einer metrisch skalierten Zielvariablen und kategorial skalierten Prädiktoren mithilfe von ANOVA (Analysis of Variance) F-Tests. Die Nullhypothese lautet: Es gibt keinen Zusammenhang zwischen der Zielvariablen und der Prädiktorvariablen. Die Berechnung eines CHAID-Dendrogramms entspricht einer Abfolge von Zusammenfügungen und Zerlegungen: In einem ersten Schritt werden die kategorialen Ausprägungsstufen jedes Prädiktors in möglichst wenige, statistisch signifikant voneinander unterschiedliche Klassen zusammengelegt. Die derart aggregierten Prädiktorvariablen werden anschließend im Hinblick auf deren statistischen Zusammenhang mit der Zielvariable in eine Rangfolge gebracht, wobei derjenige Prädiktor mit dem kleinsten $p$-Level zur Unterteilung von Ausgangs- und Unterknoten herangezogen wird (Baltes-Götz 2004).

In dieser Untersuchung bildeten die metrisch skalierten Gehalte der in den Moosen 2005 gemessenen Stoffgehalte die
Zielvariable. So erfolgte die Berechnung jeweils eines CHAIDund eines CART-Baumes für den MMI2005, der die Stoffgehalte der 12 Standardelemente zusammenfasst. Als Prädiktoren dienten die standortbeschreibenden Metainformationen sowie flächenhaft vorliegende Daten zur Umgebungsbeschreibung. Um den Verzweigungsgrad der aus den Entscheidungsbaumanalysen hervorgehenden Bäume zu begrenzen, wurde festgelegt, dass die Menge an Standorten in den zu bildenden Untergruppen nicht kleiner als 7 sein sollte, entsprechend ungefähr $1 \%$ der Größe des Ausgangsdatensatzes. Ferner wurde bei den CART-Analysen ein minimales Improvement von $1 \%$ der Ausgangsvarianz zur Bildung eines neuen Splits festgesetzt. Bei CHAID erfolgte die Festlegung eines Signifikanzniveaus von 0,01 zur Zusammenlegung der Ausgangsmenge an Prädiktorkategorien sowie zur Auswahl einer der derart aggregierten Prädiktoren für die Unterteilung des Ausgangsdatensatzes bzw. der daraus hervorgehenden Untergruppen.

\section{Ergebnisse}

\subsection{Moosproben-Sammlung}

Pleurozium schreberi ist das am häufigsten gesammelte Moos in Deutschland (1990: 70,10\%, 1995: 45,61\%, 2000: 41,25\%, 2005: 42,17), gefolgt von Scleropodium purum (1990: 16,72\%, 1995: 31,96\%, 2000: 27,72\%, 2005: 32,55\%), Нypnum cupressiforme (1990: 12,50\%, 1995: 16,37\%, 23,93\%, 2005: 24,59\%) und Hylocomium splendens (1990: 0,68\%, 1995: 5,06\%, 2000: 6,80\%, 2005: 0,55\%). Die Anteile sogenannter Fehlarten (Abietinella abietina, Brachytecium albicans, Brachytecium rutabulum, Eurhynchium praelongum, Hypnum juticulandum, Leckea polycarpa, Plagiothecium undulatum, Rhytidiadelphus squarrosus, Rhytidiadelphus triguetrus) beliefen sich auf 0\% (1990), 5,06\% (1995), $6,80 \%$ (2000) bzw. 0,55\% (2005). Diese Befunde werden in der Diskussion (Abschn. 4.1) mit den entsprechenden Zahlen zum europaweiten Monitoring-Netz 2005 verglichen.

\subsection{Qualitätskontrolle}

Im Folgenden werden Ergebnisse der Qualitätskotrolle im deutschen Moosmonitoring dargestellt. Die wesentlichen Befunde der alle europäischen Analysenergebnisse umfassenden Qualitätsprüfung werden in der Diskussion (Abschn. 4.2) behandelt. Zunächst geht es um die den deutschen Messungen zugrundeliegenden Bestimmungsgrenzen, d.h. die kleinsten Konzentrationen, die mit festgelegter Präzision für die untersuchten Metall-Elemente ermittelt werden können und oberhalb derer Analysenergebnisse quantitativ angegeben werden (Tabelle 1).

Im Hinblick auf die Vergleichbarkeit der in den Moosen bestimmten Elementgehalte in den Kampagnen 1995 und 
Tabelle 1 Bestimmungsgrenzen der im Moosmonitoring 2005 analysierten Metall-Elemente

\begin{tabular}{|c|c|c|c|c|c|c|c|c|c|c|c|c|}
\hline \multicolumn{13}{|c|}{ ICP-OES Optima 300 - Bestimmungsgrenze $[\mathrm{mg} / \mathrm{kg}]$} \\
\hline Element & $\mathrm{Al}$ & $\mathrm{Ba}$ & $\mathrm{Ca}$ & $\mathrm{Fe}$ & $\mathrm{K}$ & $\mathrm{Cu}$ & $\mathrm{Mg}$ & $\mathrm{Mn}$ & $\mathrm{Na}$ & $\mathrm{Sr}$ & $\mathrm{Ti}$ & $\mathrm{Zn}$ \\
\hline Best.grenze & 1,13 & 1 & 1,13 & 1,13 & 7,4 & 0,19 & 3,1 & 0,19 & 0,84 & 0,09 & 1 & 0,19 \\
\hline \multicolumn{13}{|c|}{ ICP-MS System 7500c - Bestimmungsgrenze $[\mathrm{mg} / \mathrm{kg}]$} \\
\hline Element & $\mathrm{Sb}$ & As & $\mathrm{Pb}$ & $\mathrm{Cd}$ & $\mathrm{Cr}$ & $\mathrm{Co}$ & Mo & $\mathrm{Ni}$ & $\mathrm{V}$ & & & \\
\hline Best.grenze & 0,003 & 0,014 & 0,002 & 0,007 & 0,010 & 0,002 & 0,002 & 0,002 & 0,003 & & & \\
\hline
\end{tabular}

PS200 Quecksilberanalysator - Bestimmungsgrenze [mg/kg]

\begin{tabular}{lc}
\hline Element & $\mathrm{Hg}$ \\
Best.grenze & 0,006
\end{tabular}

Tabelle 2 Vergleichbarkeit der Analysemethoden 2000 und 2005 anhand der Mittelwertbildung von jeweils acht Proben aus der Kampagne 2000

\begin{tabular}{lccccccc}
\hline & \multicolumn{3}{c}{ Mittelwert [mg/kg] } & \multicolumn{4}{c}{ Mittelwert [mg/kg] } \\
Element & $\mathbf{2 0 0 0}$ & $\mathbf{2 0 0 5}$ & Wfr. $^{\mathbf{1}}[\mathbf{\%}]$ & Element & $\mathbf{2 0 0 0}$ & $\mathbf{2 0 0 5}$ & Wfr. $^{\mathbf{1}}$ [\%] \\
\hline $\mathrm{Al}$ & 329 & 324 & 98,6 & $\mathrm{Mg}$ & 1264 & 1242 & 98,2 \\
$\mathrm{As}$ & 0,17 & 0,17 & 100,3 & $\mathrm{Mn}$ & 243 & 252 & 103,5 \\
$\mathrm{Ba}$ & 24,0 & 23,4 & 97,7 & $\mathrm{Na}$ & 246 & 267 & 108,0 \\
$\mathrm{Ca}$ & 3886 & 3694 & 95,1 & $\mathrm{Ni}$ & 1,42 & 1,39 & 97,7 \\
$\mathrm{Cd}$ & 0,25 & 0,26 & 102,8 & $\mathrm{~Pb}$ & 5,9 & 6,0 & 102,0 \\
$\mathrm{Cr}$ & 1,09 & 1,11 & 101,8 & $\mathrm{Sb}$ & 0,19 & 0,19 & 99,7 \\
$\mathrm{Cu}$ & 8,3 & 8,4 & 101,8 & $\mathrm{Sr}$ & 16,6 & 17,0 & 102,9 \\
$\mathrm{Fe}$ & 393 & 396 & 100,6 & $\mathrm{Ti}$ & 7,8 & 7,8 & 100,5 \\
$\mathrm{Hg}$ & 0,05 & 0,05 & 99,8 & $\mathrm{~V}$ & 1,31 & 1,33 & 102,1 \\
$\mathrm{~K}$ & 8318 & 8540 & 102,7 & $\mathrm{Zn}$ & 45 & 47 & 104,4 \\
\hline
\end{tabular}

${ }^{1}$ Wiederfindungsrate
2000 erfolgte in 2000 eine Analyse von Rückstellproben aus dem Moosmonitoring 1995 (Schröder et al. 2002). Die Ergebnisse zeigten eine sehr gute Vergleichbarkeit zwischen den Laboren der BGR und der LUFA Nord-West. Einschränkungen ergaben sich lediglich bei Elementen, die durch den gewählten Aufschluss nur unvollständig gelöst $(\mathrm{Al}, \mathrm{Cr}, \mathrm{Ti})$ oder deren Analyse im Nachweisbereich mit unvermeidbaren Streuungen behaftet ist $(\mathrm{Hg})$.

Zur Quantifizierung der Vergleichbarkeit von Ergebnissen der Kampagnen 2000 und 2005 wurden acht Moosproben aus dem vorhandenen Rückstellmuster aus der Kampagne 2000 ausgewählt und auf 20 Elemente untersucht. Zum Vergleich wurden die Analysenergebnisse der acht Proben vom Jahr 2000 gemittelt und die daraus resultierenden Werte als $100 \%$ gesetzt. Die entsprechend abgeleiteten Mittelwerte der Kampagne 2005 wurden anschließend als prozentuale Wiederfindungsraten der in der Kampagne 2000 gemessenen Werte berechnet. Zwei Messwerte $(1 \times \mathrm{Na}$ und $1 \times \mathrm{Al})$ wurden als Ausreißer aus den Analysen von 2000 eliminiert. Die Wiederfindungsraten liegen zwischen $95 \%$ und 108\% (Tabelle 2).

Die Analysen der Referenzproben M2 und M3 zeigen eine gute bis sehr gute Übereinstimmung mit den Soll-Werten (Tabelle 3). Die Unterschiede in der Trocknungstemperatur gegenüber der Kampagne $2000\left(=40^{\circ} \mathrm{C}\right)$ ist im Hinblick auf die Vergleichbarkeit der Hg-Bestimmung nicht problematisch. Metallisches Hg ist bei Zimmertemperatur flüchtig und seine quantitative Bestimmung entsprechend der hier verwendeten Methode schwer möglich. Die Wiederfindung von metallischem Hg in Referenz- oder Rückstellproben, die nicht eingefroren sind, ist somit sehr schlecht. Was in den Umweltproben analysiert wird, sind die verschiedenen Hg-Salze bzw. -Oxide, die erst bei höheren Temperaturen verdampfen. Die Trocknung bei $70^{\circ} \mathrm{C}$ hat somit keinen Einfluss auf den $\mathrm{Hg}$-Gehalt der Moosproben.

In der Kampagne 2000 erfolgte ein Vergleich einer Scheibenschwingmühle mit Achatmahlgarnitur und der 2005 verwendeten Tecator-Mühle hinsichtlich des Abriebs der Elemente Al, Fe, Kobalt (Co), Cr, Molybdän (Mo), Ni und V. Die Kontamination der Proben durch Abrieb war insgesamt sehr gering, bei der Tercator-Mühle sogar noch geringer als bei der Scheibenschwingmühle (Schröder et al. 2002).

Bei jeder Messserie wurden jeweils eine M2- und eine M3-Referenzprobe mit in die Analysen einbezogen und die daraus resultierenden Messwerte dokumentiert (Tabelle 4). Die Wiederfindungsrate für die Moosreferenz M2 beträgt zwischen $78 \%(\mathrm{Sb})$ und $110 \%(\mathrm{~K}$ und $\mathrm{Na}$ ) sowie für die Moosreferenz M3 zwischen 69\% (Mo) und 121\% (Ni). 
Tabelle 3 Vergleich der Referenzwerte nach Steinnes et al. (1997) mit den Ergebnissen im Moosmonitoring 2005

\begin{tabular}{|c|c|c|c|c|c|c|c|c|c|c|c|c|}
\hline \multirow{3}{*}{ Element } & \multicolumn{5}{|c|}{ Moosreferenzmaterial M 2} & & \multicolumn{6}{|c|}{ Moosreferenzmaterial M 3} \\
\hline & \multirow[t]{2}{*}{$\mathbf{n}$} & \multicolumn{2}{|c|}{ Steinnes et al. (1997) } & \multicolumn{2}{|c|}{ Moosmonit. 2005} & & \multirow[t]{2}{*}{ Element } & \multirow[t]{2}{*}{$\mathbf{n}$} & \multicolumn{2}{|c|}{ Steinnes et al. (1997) } & \multicolumn{2}{|c|}{ Moosmonit. 2005} \\
\hline & & $\begin{array}{c}\text { Mw } \\
{[\mathrm{mg} / \mathrm{kg}]}\end{array}$ & $\begin{array}{c}\text { Stabw } \\
{[\mathrm{mg} / \mathrm{kg}]}\end{array}$ & $\begin{array}{c}\text { Mw } \\
{[\mathrm{mg} / \mathrm{kg}]}\end{array}$ & $\begin{array}{c}\text { Stabw } \\
{[\mathrm{mg} / \mathrm{kg}]}\end{array}$ & & & & $\begin{array}{c}\text { Mw } \\
{[\mathrm{mg} / \mathrm{kg}]}\end{array}$ & $\begin{array}{c}\text { Stabw } \\
{[\mathrm{mg} / \mathrm{kg}]}\end{array}$ & $\begin{array}{c}\text { Mw } \\
{[\mathrm{mg} / \mathrm{kg}]}\end{array}$ & $\begin{array}{l}\text { Stabw } \\
{[\mathrm{mg} / \mathrm{kg}]}\end{array}$ \\
\hline As & 14 & 0,98 & 0,07 & 0,94 & 0,08 & As & & 11 & 0,105 & 0,007 & 0,12 & 0,01 \\
\hline Cd & 15 & 0,45 & 0,02 & 0,46 & 0,03 & & Cd & 17 & 0,106 & 0,005 & 0,11 & 0,01 \\
\hline $\mathrm{Cr}$ & 21 & 0,97 & 0,17 & 0,83 & 0,09 & & $\mathrm{Cr}$ & 25 & 0,67 & 0,19 & 0,53 & 0,09 \\
\hline $\mathrm{Cu}$ & 18 & 68,7 & 2,5 & 69,3 & 2,5 & & $\mathrm{Cu}$ & 20 & 3,76 & 0,23 & 3,6 & 0,1 \\
\hline $\mathrm{Fe}$ & 29 & 262 & 35 & 274 & 18 & & $\mathrm{Fe}$ & 18 & 138 & 12 & 146 & 8 \\
\hline Hg & 15 & 0,058 & 0,05 & 0,052 & 0,002 & & $\mathrm{Hg}$ & 11 & 0,035 & 0,004 & 0,038 & 0,002 \\
\hline $\mathbf{N i}$ & 19 & 16,3 & 0,9 & 15,15 & 1,05 & & $\mathrm{Ni}$ & 15 & 0,95 & 0,08 & 1,09 & 0,14 \\
\hline $\mathbf{P b}$ & 15 & 6,37 & 0,43 & 6,8 & 0,5 & & $\mathbf{P b}$ & 19 & 3,33 & 0,25 & 3,4 & 0,2 \\
\hline Sb & 5 & 0,21 & 0,016 & 0,18 & 0,01 & & Sb & 4 & 0,052 & 0,007 & 0,05 & 0,01 \\
\hline $\mathbf{V}$ & 20 & 1,43 & 0,17 & 1,33 & 0,11 & & $\mathbf{V}$ & 19 & 1,19 & 0,15 & 1,12 & 0,06 \\
\hline $\mathrm{Zn}$ & 17 & 36,1 & 1,2 & 38,4 & 2,4 & & $\mathrm{Zn}$ & 21 & 25,4 & 1,1 & 25,7 & 0,8 \\
\hline Al & 6 & 178 & 15 & 184 & 20 & & Al & 5 & 169 & 10 & 161 & 13 \\
\hline $\mathbf{B a}$ & 8 & 17,6 & 0,7 & 17,1 & 0,4 & & $\mathbf{B a}$ & 9 & 13,7 & 0,6 & 13,5 & 0,8 \\
\hline $\mathrm{Ca}$ & 5 & 1910 & 100 & 2108 & 65 & & $\mathrm{Ca}$ & 5 & 1920 & 70 & 2213 & 54 \\
\hline Co & 8 & 0,98 & 0,06 & 0,87 & 0,08 & & Co & 7 & 0,115 & 0,006 & 0,11 & 0,01 \\
\hline $\mathbf{K}$ & 4 & 6980 & 350 & 7891 & 162 & & $\mathbf{K}$ & 5 & 4510 & 280 & 5291 & 125 \\
\hline Mg & 6 & 826 & 52 & 796 & 25 & & Mg & 8 & 755 & 77 & 783 & 27 \\
\hline Mn & 15 & 342 & 17 & 335 & 12 & & Mn & 14 & 535 & 30 & 542 & 19 \\
\hline Mo & 5 & 0,23 & 0,04 & 0,26 & 0,03 & & Mo & 5 & 0,1 & 0,04 & 0,07 & 0,02 \\
\hline $\mathrm{Na}$ & 6 & 166 & 15 & 193 & 23 & & $\mathrm{Na}$ & 5 & 133 & 12 & 130 & 14 \\
\hline $\mathrm{Sr}$ & 4 & 5,31 & 0,15 & 5,6 & 0,6 & & $\mathrm{Sr}$ & 6 & 4,64 & 0,24 & 4,9 & 0,2 \\
\hline
\end{tabular}

Tabelle 4 Ergebnisse der Qualitätskontrollen im Moosmonitoring 2005 anhand der Referenzmaterialien M2 und M3 (Steinnes et al. 1997)

\begin{tabular}{|c|c|c|c|c|c|c|c|c|c|c|c|}
\hline \multirow{3}{*}{ Element } & \multicolumn{4}{|c|}{ Moosreferenzmaterial M 2} & & \multicolumn{6}{|c|}{ Moosreferenzmaterial M 2} \\
\hline & \multicolumn{2}{|c|}{ Steinnes et al. (1997) } & \multicolumn{3}{|c|}{ Moosmonit. 2005} & \multirow[t]{2}{*}{ Element } & \multicolumn{2}{|c|}{ Steinnes et al. (1997) } & \multicolumn{3}{|c|}{ Moosmonit. 2005} \\
\hline & $\begin{array}{c}\text { Soll } \\
{[\mathrm{mg} / \mathrm{kg}]}\end{array}$ & $\begin{array}{l}\text { Stabw. } \\
\text { [mg/kg] }\end{array}$ & $\mathbf{n}$ & $\begin{array}{c}\text { Mw } \\
{[\mathrm{mg} / \mathrm{kg}]}\end{array}$ & $\begin{array}{c}\text { Wfr. }{ }^{1} \\
{[\%]}\end{array}$ & & $\begin{array}{c}\text { Soll } \\
{[\mathrm{mg} / \mathrm{kg}]}\end{array}$ & $\begin{array}{l}\text { Stabw. } \\
{[\mathrm{mg} / \mathrm{kg}]}\end{array}$ & $\mathbf{n}$ & $\begin{array}{c}\text { Mw } \\
{[\mathrm{mg} / \mathrm{kg}]}\end{array}$ & $\begin{array}{l}\text { Wfr. }{ }^{1} \\
{[\%]}\end{array}$ \\
\hline Al & 178 & 15 & 16 & 179 & 101 & Al & 169 & 10 & 16 & 163 & 96 \\
\hline As & 0,98 & 0,07 & 16 & 0,88 & 90 & As & 0,105 & 0,007 & 16 & 0,123 & 117 \\
\hline $\mathbf{B a}$ & 17,6 & 0,7 & 16 & 16,5 & 94 & $\mathbf{B a}$ & 13,7 & 0,6 & 16 & 12,4 & 91 \\
\hline $\mathrm{Ca}$ & 1910 & 100 & 16 & 2064 & 108 & $\mathrm{Ca}$ & 1920 & 70 & 16 & 222 & 116 \\
\hline Cd & 0,45 & 0,02 & 16 & 0,44 & 97 & Cd & 0,106 & 0,005 & 16 & 0,111 & 104 \\
\hline Co & 0,98 & 0,06 & 16 & 0,84 & 86 & Co & 0,115 & 0,006 & 16 & 0,114 & 100 \\
\hline $\mathrm{Cr}$ & 0,97 & 0,17 & 16 & 0,82 & 85 & $\mathrm{Cr}$ & 0,67 & 0,19 & 16 & 0,57 & 85 \\
\hline $\mathrm{Cu}$ & 68,7 & 2,3 & 16 & 67,4 & 98 & $\mathrm{Cu}$ & 3,76 & 0,23 & 16 & 3,87 & 103 \\
\hline $\mathrm{Fe}$ & 262 & 35 & 16 & 266 & 102 & $\mathrm{Fe}$ & 138 & 12 & 16 & 150 & 108 \\
\hline $\mathrm{Hg}$ & 0,058 & 0,005 & 16 & 0,049 & 85 & Hg & 0,035 & 0,004 & 16 & 0,036 & 103 \\
\hline $\mathbf{K}$ & 6980 & 350 & 16 & 7664 & 110 & $\mathbf{K}$ & 4510 & 280 & 16 & 5207 & 116 \\
\hline Mg & 826 & 52 & 16 & 783 & 95 & Mg & 755 & 77 & 16 & 783 & 104 \\
\hline Mn & 342 & 17 & 16 & 329 & 96 & Mn & 535 & 30 & 16 & 532 & 100 \\
\hline Mo & 0,23 & 0,04 & 16 & 0,21 & 93 & Mo & 0,1 & 0,04 & 16 & 0,07 & 69 \\
\hline $\mathrm{Na}$ & 166 & 15 & 16 & 183 & 110 & $\mathrm{Na}$ & 133 & 12 & 16 & 131 & 98 \\
\hline $\mathbf{N i}$ & 16,3 & 0,9 & 16 & 14,7 & 90 & $\mathrm{Ni}$ & 0,95 & 0,08 & 16 & 1,15 & 121 \\
\hline $\mathbf{P b}$ & 6,37 & 0,43 & 16 & 6,63 & 104 & $\mathbf{P b}$ & 3,33 & 0,25 & 16 & 3,56 & 107 \\
\hline $\mathbf{S}$ & 963 & 93 & 16 & 929 & 96 & $\mathbf{S}$ & 830 & 74 & 16 & 831 & 100 \\
\hline Sb & 0,21 & 0,016 & 16 & 0,16 & 78 & $\mathrm{Sb}$ & 0,052 & 0,007 & 16 & 0,04 & 77 \\
\hline $\mathrm{Sr}$ & 5,3 & 0,15 & 16 & 5,3 & 100 & $\mathrm{Sr}$ & 4,6 & 0,24 & 16 & 4,75 & 102 \\
\hline $\mathbf{T i}$ & - & - & 16 & 12,1 & - & $\mathbf{T i}$ & - & - & 16 & 8,7 & - \\
\hline $\mathbf{V}$ & 1,43 & 0,17 & 16 & 1,28 & 89 & $\mathbf{V}$ & 1,19 & 0,15 & 16 & 1,15 & 97 \\
\hline $\mathrm{Zn}$ & 36,1 & 1,2 & 16 & 35,8 & 99 & $\mathrm{Zn}$ & 25,4 & 1,1 & 16 & 25,8 & 102 \\
\hline
\end{tabular}

${ }^{1}$ Wiederfindungsrate 
Tabelle 5 Ergebnisse grenzüberschreitender Qualitätskontrollen für Belgien, Deutschland, Frankreich, Österreich, Polen, Schweiz und Tschechien

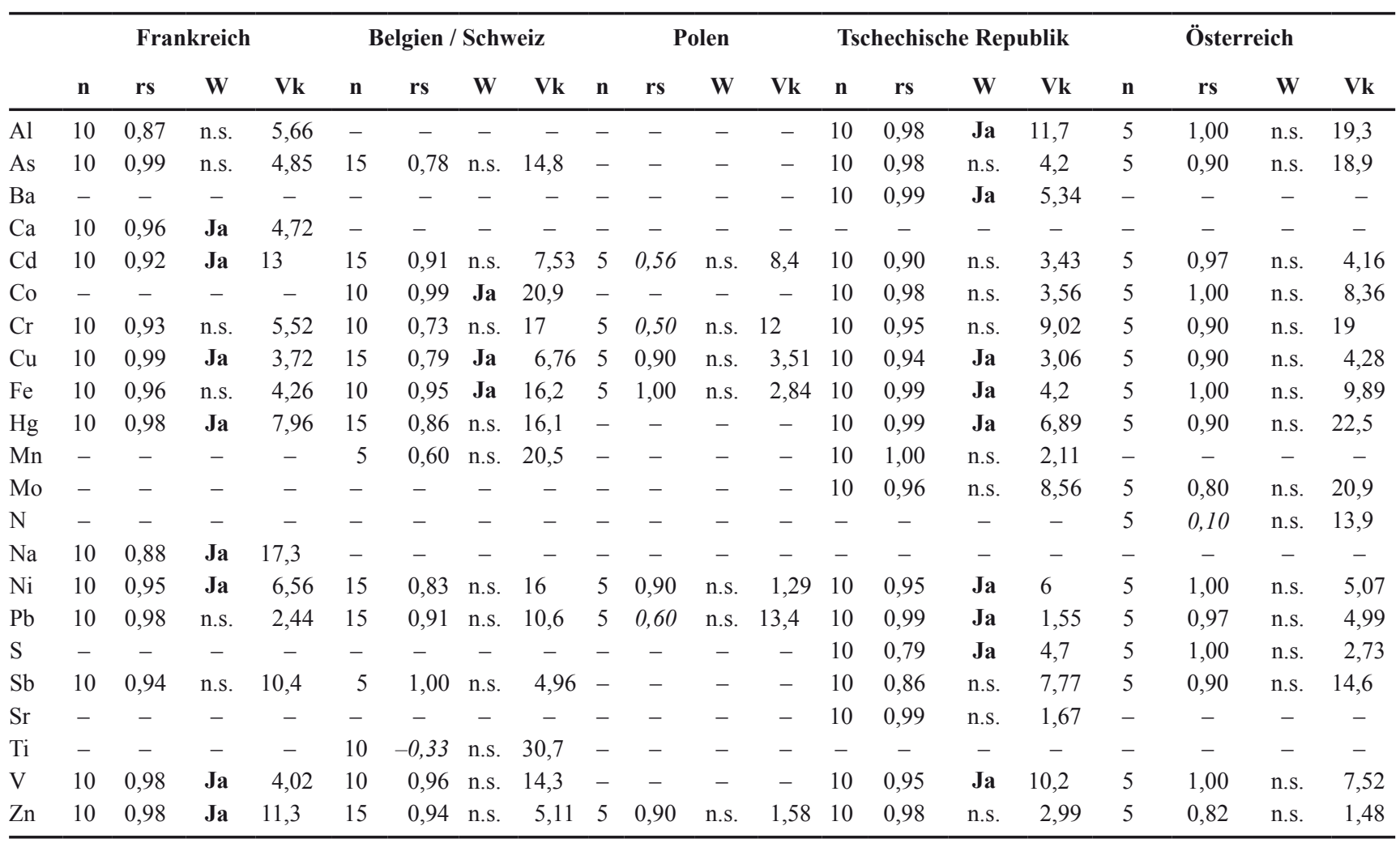

rs Korrelation Koeffizient nach Spearman $(\mathrm{p}<0,05)$

W Signifikante Unterschiede? ( $<<0,05 ; \mathrm{Ja} / \mathrm{n} . \mathrm{s}$.)

Vk Durchschnittliche Variationskoeffizienten [\%]

Mit den an Deutschland angrenzenden, am UNECE Moosmonitoring teilnehmenden Staaten Belgien, Frankreich, Schweiz, Österreich, Tschechien sowie Polen wurde ein Laborvergleich auf Basis von fünf Proben aus dem jeweils grenznahen Raum vereinbart. Die Analysen für die Schweiz und aus Belgien wurden von einem Labor durchgeführt. Die Ergebnisse der fünf Laborvergleiche in Form korrelations- und inferenzstatistischer Statistiken sind der Tabelle $5 \mathrm{zu}$ entnehmen. Angegeben sind ferner die über die jeweiligen Messwertvergleiche ermittelten mittleren Variationskoeffizienten sowie der Anteil an Messergebnissen, bei denen im Labor des jeweiligen Anrainerstaats höhere Werte gemessen wurden als im deutschen Labor.

Im Hinblick auf den Vergleich Frankreich - Deutschland sind bei allen Elementen hohe, statistisch signifikante Korrelationen oberhalb von 0,87 festzuhalten. Die mittleren Variationskoeffizienten deuten mit maximal $17 \%$ auf größtenteils geringe Unterschiede hin. Allerdings existieren signifikante Unterschiede bei den Elementen $\mathrm{Ca}, \mathrm{Cd}, \mathrm{Cu}, \mathrm{Hg}, \mathrm{Na}, \mathrm{Ni}, \mathrm{V}$ und $\mathrm{Zn}$. Bei diesen Elementen zeigen sich dann auch durchgängig höhere gemessene Werte im Labor der LUFA Nord-West (Ca, $\mathrm{Cd}, \mathrm{Cu}, \mathrm{Zn})$ bzw. im französischen Labor (Hg, Na, Ni, V).

Der Vergleich Belgien - Schweiz - Deutschland zeigt größtenteils gute Übereinstimmungen beider Labore. Die Korrela- tionskoeffizienten liegen meist oberhalb von 0,75 und sind bis auf die Ausnahme Ti auch statistisch signifikant. Bei Ti zeigen sich auch die größten mittleren prozentualen Abweichungen, obwohl keine statistisch signifikanten Unterschiede festgestellt werden können. Statistisch signifikante Unterschiede zeigen sich lediglich bei den Elementen $\mathrm{Co}, \mathrm{Cu}$ und $\mathrm{Fe}$.

Beim Vergleich der Labore Polen - Deutschland liegen bei Berücksichtigung der niedrigen Fallzahlen keine signifikanten Unterschiede vor. Die mittleren Schwankungen zwischen den in beiden Laboren gemessenen Messwerten liegen maximal bei $13 \%(\mathrm{~Pb})$. Allerdings zeigen die Ergebnisse der Korrelationsanalysen nach Spearman z.T. nicht signifikante Korrelationen auf.

Der Vergleich Tschechien - Deutschland zeigt bis auf eine Ausnahme (S) hohe signifikante Korrelationen der in beiden Laboren gemessenen Werte, äußerst niedrige mittlere Variationskoeffizienten mit maximal $10 \%(\mathrm{~V})$ und $12 \%(\mathrm{Al})$, allerdings signifikante Unterschiede in Bezug auf die neun Elemente $\mathrm{Al}, \mathrm{Ba}, \mathrm{Cu}, \mathrm{Fe}, \mathrm{Hg}, \mathrm{Ni}, \mathrm{Pb}, \mathrm{S}$ und V. Hierbei zeigen $\mathrm{Al}, \mathrm{Ba}, \mathrm{Fe}, \mathrm{Hg}, \mathrm{Ni}, \mathrm{S}$ und $\mathrm{V}$ ausschließlich bzw. größtenteils höhere Messwerte im tschechischen Labor, bei den übrigen Elementen $\mathrm{Cu}$ und $\mathrm{Pb}$ liegt der umgekehrte Fall vor.

Der auf insgesamt fünf Moosproben aufbauende Laborvergleich zwischen Österreich und Deutschland zeigt bis auf N und 
Tabelle 6 Z-Scores des Internationalen Ringversuchs „Wageningen“ Juli-September 2006

\begin{tabular}{lcccc}
\hline Probe & Tulpe & Gras & Mais & Schilfrohr \\
\hline Ca & 0,24 & $-0,57$ & 1,07 & 0,45 \\
Cd & 0,77 & $-0,20$ & 1,35 & $-0,22$ \\
Co & 0,36 & $-0,18$ & 1,45 & $-1,13$ \\
Cr & - & $-0,32$ & $-\mathbf{4 , 6 0}$ & $-0,21$ \\
Cu & 0,21 & 0,11 & 0,73 & 0,14 \\
Fe & 1,32 & 0,22 & 0,86 & 0,81 \\
Hg & $-0,28$ & 0,59 & 2,54 & $-0,37$ \\
K & 0,92 & 0,16 & 1,70 & 0,37 \\
Mg & $-0,07$ & $-1,42$ & 0,06 & $-0,13$ \\
Mo & 0,15 & $-0,73$ & $-0,05$ & $-0,73$ \\
Na & $-1,17$ & $-1,01$ & $-0,77$ & 0,04 \\
Ni & $-0,89$ & $-1,15$ & 0,70 & 0,34 \\
P & 0,68 & $-0,23$ & 0,70 & $-0,27$ \\
S & 0,17 & 0,07 & 0,37 & 0,26 \\
V & - & - & 0,70 & $-0,63$ \\
Zn & 0,79 & 0,06 & 1,11 & 0,80 \\
\hline
\end{tabular}

Zn hohe signifikante Korrelationen oberhalb von 0,9 auf. Es können keine statistisch signifikanten Unterschiede beider Messwertreihen festgestellt werden, wobei die mittleren Variationskoeffizienten außer bei $\mathrm{Hg}$ und Mo unterhalb von $20 \%$ liegen.

Berücksichtigt man die z. T. äußerst niedrigen Fallzahlen, deutet der Laborvergleich auf gute Übereinstimmung zwischen den beteiligten Laboren hin. Der einzige auffällige Unterschied liegt bei Ti im Vergleich zum belgischen Labor (prozentualer Unterschied $=31 \%$, keine statistisch signifikante Korrelation) vor. In einigen Fällen können zwar signifikante Unterschiede festgestellt werden, jedoch liegen die damit in Verbindung stehenden prozentualen Unterschiede zumeist unterhalb von $10 \%$.

Diese Befunde werden durch die Ergebnisse des Internationalen Ringversuchs „Wageningen“ für die Periode Juli-September 2006 bestätigt (Tabelle 6). Es zeigt sich, dass über alle 61 Z-Scores nur zwei Ausreißer (Z-Score > 2, $<-2$ ) vorhanden sind, was eine sehr gute Untersuchungsqualität der Laboranalysen belegt.

Die von 2000 nach 2005 angestiegenen Cr-Werte gaben Anlass zu einer weiteren externen Qualitätskontrolle durch das Landesamt für Natur, Umwelt und Verbraucherschutz Nordrhein-Westfalens (LANUV). Auch diese Überprüfung bestätigte die gute Analysenqualität im Moosmonitoring. Dies sowie die Ergebnisse der internen (siehe Tabellen 1, 4) und laborexternen (siehe Tabellen 5, 6) Qualitätskontrolle belegen, dass die Ergebnisse des Moosmonitorings Staaten und Messkampagnen übergreifend gut vergleichbar sind. Dies ist die entscheidende Voraussetzung dafür, räumliche und zeitliche Messwertunterschiede zum Gegenstand statistischer Auswertungen zu machen. Diese Ergebnisse werden in der Diskussion (Abschnit 4.2) mit den entsprechenden Zahlen des europaweiten Messnetzes 2005 verglichen.
Tabelle 7 Geostatistische Kenngrößen Moosmonitoring 2005

\begin{tabular}{lllrr}
\hline Element & $\mathbf{R}$ & N/S & ME & MPE \\
\hline $\mathrm{As}$ & 138 & 0,71 & 0,00 & 26,9 \\
$\mathrm{Cd}$ & 145 & 0,76 & 0,00 & 18,9 \\
$\mathrm{Cr}$ & 123 & 0,61 & $-0,23$ & 40,4 \\
$\mathrm{Cu}$ & 132 & 0,52 & 0,00 & 14,3 \\
$\mathrm{Fe}$ & 121 & 0,68 & $-4,95$ & 23,0 \\
$\mathrm{Hg}$ & 142 & 0,72 & 0,00 & 14,1 \\
$\mathrm{Ni}$ & 174 & 0,7 & 0,00 & 23,4 \\
$\mathrm{~Pb}$ & 153 & 0,78 & $-0,06$ & 27,7 \\
$\mathrm{Ti}$ & 175 & 0,81 & $-0,34$ & 30,8 \\
$\mathrm{Sb}$ & 170 & 0,63 & 0,00 & 24,2 \\
$\mathrm{~V}$ & 106 & 0,79 & 0,00 & 19,5 \\
$\mathrm{Zn}$ & 133 & 0,64 & $-0,20$ & 19,8 \\
$\mathrm{~N}$ & 84 & 0,68 & 0,01 & 9,4 \\
\hline
\end{tabular}

R Reichweite der räumlichen Autokorrelation (Einheit: km) N/S Verhältnis von Nugget-Effekt zu Sill

ME Mean Error (Mittlerer Fehler - Metalle $[\mu \mathrm{g} / \mathrm{g}]$, N\% Tr.)

MPE Median of Percental Errors (Median der Prozentualen Fehler) multipliziert mit dem Verhältnis der Spannweiten von Kreuzvalidierungsfehlern und empirischen Messwerten (Einheit: \%)

\subsection{Plausibilitätskontrolle}

Die deutschen Plausibilitätsprüfungen legten es nahe, 12 Messwerte aus Nordrhein-Westfallen (NW11 - Cd), NW17 - Zn, NW29 - As, NW30 - Ba, Cu, Pb, NW31 - Cd, NW36 - N, NW65 - Sb, NW63 - Cr, NW70 - Zn, NW86 - Na), einen kompletten Standortdatensatz (BY121) und zwei einzelne Messwerte (BY217 - Ni, BY49 - Zn) aus Bayern sowie einen kompletten Standortdatensatz aus Sachsen-Anhalt (ST203) bei den weiteren statistischen Analysen nicht weiter zu berücksichtigen. Diese Befunde werden in der Diskussion (Abschn. 4.3) mit den Erkenntnissen über das Europa-Messnetz 2005 verglichen.

\subsection{Geostatistik}

Die Kenngrößen der geostatistischen Analysen und der Kreuzvalidierung (Tabelle 7) zeigen zwei Maßstabsbereiche der räumlichen Variation. Zum einen handelt es sich um Varianzen bei Messpunktabständen unterhalb des einfachen mittleren Messpunktabstands $(=15,7 \mathrm{~km}=$ erste Distanzklasse), die durch die Nugget-Varianz quantifiziert und fortan als kleinräumige, eher lokale oder standortspezifische Varianzen bezeichnet werden. Zum anderen lassen sich Messwertunterschiede identifizieren, deren räumliche Dimension durch die Autokorrelationsreichweiten, den Range ( $\mathrm{R}$ in Tabelle 7), und deren Ausmaß durch den Sill (S) beziffert werden. Das Verhältnis zwischen den kleinräumigen, eher lokalen Varianzen und den großräumigen, regionalen Messwertunterschieden wird mit dem Nugget/Sill-Wert (N/S in Tabelle 7) gemessen. Demnach beträgt der Anteil der kleinräumigen Varianz an 
Tabelle 8 Zeitliche Entwicklung des 20., 50., und 90. Perzentils der Akkumulation von Metallen in Moosen sowie des MMI

\begin{tabular}{|c|c|c|c|c|}
\hline \multicolumn{5}{|c|}{ 20. Percentil } \\
\hline & 1990 & 1995 & 2000 & 2005 \\
\hline As & 0,222 & 0,150 & 0,110 & 0,110 \\
\hline Cd & 0,218 & 0,230 & 0,160 & 0,150 \\
\hline $\mathrm{Cr}$ & 1,53 & 0,93 & 0,69 & 1,41 \\
\hline $\mathrm{Cu}$ & 6,62 & 7,27 & 5,56 & 5,72 \\
\hline $\mathrm{Fe}$ & 380 & 311 & 236 & 236 \\
\hline Hg & - & 0,0300 & 0,0310 & 0,0271 \\
\hline $\mathbf{N i}$ & 1,69 & 1,16 & 0,83 & 0,82 \\
\hline $\mathbf{P b}$ & 10,1 & 5,37 & 3,09 & 2,47 \\
\hline Sb & & 0,125 & 0,110 & 0,110 \\
\hline $\mathbf{T i}$ & 20,9 & 14,8 & 5,13 & 4,33 \\
\hline $\mathbf{V}$ & 2,32 & 1,22 & 0,74 & 0,75 \\
\hline Zn & 35,2 & 42,2 & 32,3 & 34,7 \\
\hline $\mathbf{M M I}_{1990-2005}$ & 6 & 4,88 & 2,25 & 2,88 \\
\hline MMI $_{1995-2005}$ & & 5,25 & 2,75 & 3,17 \\
\hline \multicolumn{5}{|c|}{ 50. Percentil } \\
\hline & 1990 & 1995 & 2000 & 2005 \\
\hline As & 0,338 & 0,249 & 0,160 & 0,160 \\
\hline Cd & 0,287 & 0,293 & 0,210 & 0,210 \\
\hline $\mathrm{Cr}$ & 2,20 & 1,41 & 0,91 & 2,36 \\
\hline $\mathrm{Cu}$ & 8,79 & 9,45 & 7,14 & 7,27 \\
\hline $\mathbf{F e}$ & 618 & 446 & 343 & 328 \\
\hline Hg & - & 0,0420 & 0,0410 & 0,0353 \\
\hline $\mathbf{N i}$ & 2,35 & 1,63 & 1,13 & 1,16 \\
\hline $\mathbf{P b}$ & 12,9 & 7,78 & 4,62 & 3,69 \\
\hline Sb & - & 0,173 & 0,150 & 0,160 \\
\hline $\mathbf{T i}$ & 27,8 & 21,8 & 7,94 & 7,11 \\
\hline $\mathbf{V}$ & 3,10 & 1,75 & 1,06 & 1,09 \\
\hline $\mathrm{Zn}$ & 43,5 & 53,7 & 41,0 & 46,7 \\
\hline MMI $_{1990-2005}$ & 7,625 & 6,50 & 3,88 & 4,38 \\
\hline MMI $_{1995-2005}$ & & 6,75 & 4,50 & 4,83 \\
\hline
\end{tabular}

\begin{tabular}{|c|c|c|c|c|}
\hline \multicolumn{5}{|c|}{ 90. Percentil } \\
\hline & 1990 & 1995 & 2000 & 2005 \\
\hline As & 0,607 & 0,597 & 0,380 & 0,380 \\
\hline Cd & 0,491 & 0,505 & 0,375 & 0,370 \\
\hline $\mathrm{Cr}$ & 4,23 & 3,13 & 1,62 & 7,11 \\
\hline $\mathrm{Cu}$ & 13,08 & 14,50 & 10,55 & 11,09 \\
\hline $\mathrm{Fe}$ & 1438 & 901 & 661 & 713 \\
\hline Hg & - & 0,0773 & 0,0710 & 0,0561 \\
\hline $\mathbf{N i}$ & 4,47 & 3,02 & 2,21 & 2,33 \\
\hline $\mathbf{P b}$ & 23,5 & 15,73 & 9,24 & 7,63 \\
\hline Sb & - & 0,300 & 0,260 & 0,280 \\
\hline $\mathbf{T i}$ & 78,6 & 47,0 & 18,31 & 18,55 \\
\hline $\mathbf{V}$ & 5,20 & 3,32 & 1,96 & 1,87 \\
\hline $\mathbf{Z n}$ & 69,9 & 86,5 & 64,5 & 82,3 \\
\hline MMI $_{1990-2005}$ & 9,25 & 8,75 & 6,63 & 7,13 \\
\hline MMI $_{1995-2005}$ & & 8,86 & 7,42 & 7,58 \\
\hline
\end{tabular}

derjenigen Varianz, die großräumlich, d.h. in der Reichweite räumlicher Autokorrelation mit Werten von $84 \mathrm{~km}$ bis $175 \mathrm{~km}$ erreicht wird, zwischen $52 \%$ und $81 \%$. Die Ergebnisse der Kreuzvalidierung belegen eine ausreichende Präzision der Flächenschätzungen. Folglich wurden für die Elemente As, $\mathrm{Cd}, \mathrm{Cr}, \mathrm{Cu}, \mathrm{Fe}, \mathrm{Hg}, \mathrm{Ni}, \mathrm{Pb}, \mathrm{Sb}, \mathrm{Ti}, \mathrm{V}$ und $\mathrm{Zn}$ flächenhafte Karten geostatistisch berechnet, welche die Grundlage für die Karten der mit dem MMI1995-2005 zusammenfassend beschriebenen Metall-Bioakkumulation bilden (Abschn. 3.5).

\subsection{Zeitliche Entwicklung}

der elementspezifischen Bioakkumulation

und der Multi-Metall-Indizes (MMI)

Die Perzentilstatistik der gemessenen Metalle und der zusammenfassenden MMI ermöglichen einen Überblick der zeitlichen Entwicklung der Bioakkumulation (Tabelle 8). Anhand des 20., 50. und 90. Perzentils sowie des MMI lässt sich eine deutliche, zumeist kontinuierliche Abnahme der Metallanreicherung in Moosen zwischen 1990 und 2000 nachweisen. Dieser Trend setzt sich nicht bei allen Metallen bis 2005 fort. Bei den 20. Perzentilen stiegen die Werte von $\mathrm{Cd}, \mathrm{Hg}, \mathrm{Ni}, \mathrm{Pb}$, Ti nicht an. Dasselbe gilt für die 50. Perzentile, die Mediane, von $\mathrm{Fe}, \mathrm{Hg}, \mathrm{Pb}$ und $\mathrm{Ti}$ sowie für die 90 . Perzentile von $\mathrm{Cd}$, $\mathrm{Hg}, \mathrm{Pb}$, und $\mathrm{V}$. Mithilfe von Mann-Whitney-U-Tests berechnete Irrtumswahrscheinlichkeiten ergaben von 2000 nach 2005 signifikante Zunahmen ( $\mathrm{p}<0,05)$ von $\mathrm{Cr}(159 \%), \mathrm{Cu}$ (2\%), Sb (7\%) und $\mathrm{Zn}(14 \%)$ sowie signifikante Abnahmen $(\mathrm{p}<0,05)$ von $\mathrm{Hg}(14 \%), \mathrm{Pb}(20 \%)$ und $\mathrm{Ti}(10 \%)$. Beide MMI steigen von 2000 nach 2005 um etwa 0,5 Stufen auf 7,13 (MMI1990-2005) bzw. 7,58 (MMI1995-2005) an.

Die räumliche Differenzierung der in Tabelle 8 zusammenfassend beschriebenen zeitlichen Entwicklung der Metallanreicherung wird am Beispiel des MMI1995-2005 kartografisch veranschaulicht (Abb. 1).

\subsection{Zusammenhänge der Bioakkumulation} mit Deposition sowie Eigenschaften der Moossammelorte und ihrer Umgebung

\subsubsection{Bivariate Korrelationsanalyse}

Viele der signifikanten statistischen Zusammenhänge zwischen den Konzentrationen von jeweils zwei Metallen (Tabelle 9) rangieren zwischen 0,5 und 0,9. Jedoch erweisen sich diese Zusammenhänge als zeitlich nicht stabil, so betragen beispielsweise die Korrelationen zwischen $\mathrm{Cd}$ und Cr 0,07 (1990), 0,7 (1995, 2000) und 0,26 (2005).

Die Rankkorrelationen zwischen den Metallkonzentrationen in Moosen einerseits und der Deposition dieser Elemente innerhalb und außerhalb von Wäldern (Tabelle 10) sowie modellierter Nass-, Trocken- und Gesamtdeposition andererseits (Abb. 2) variieren nach Stoffen und Depo- 

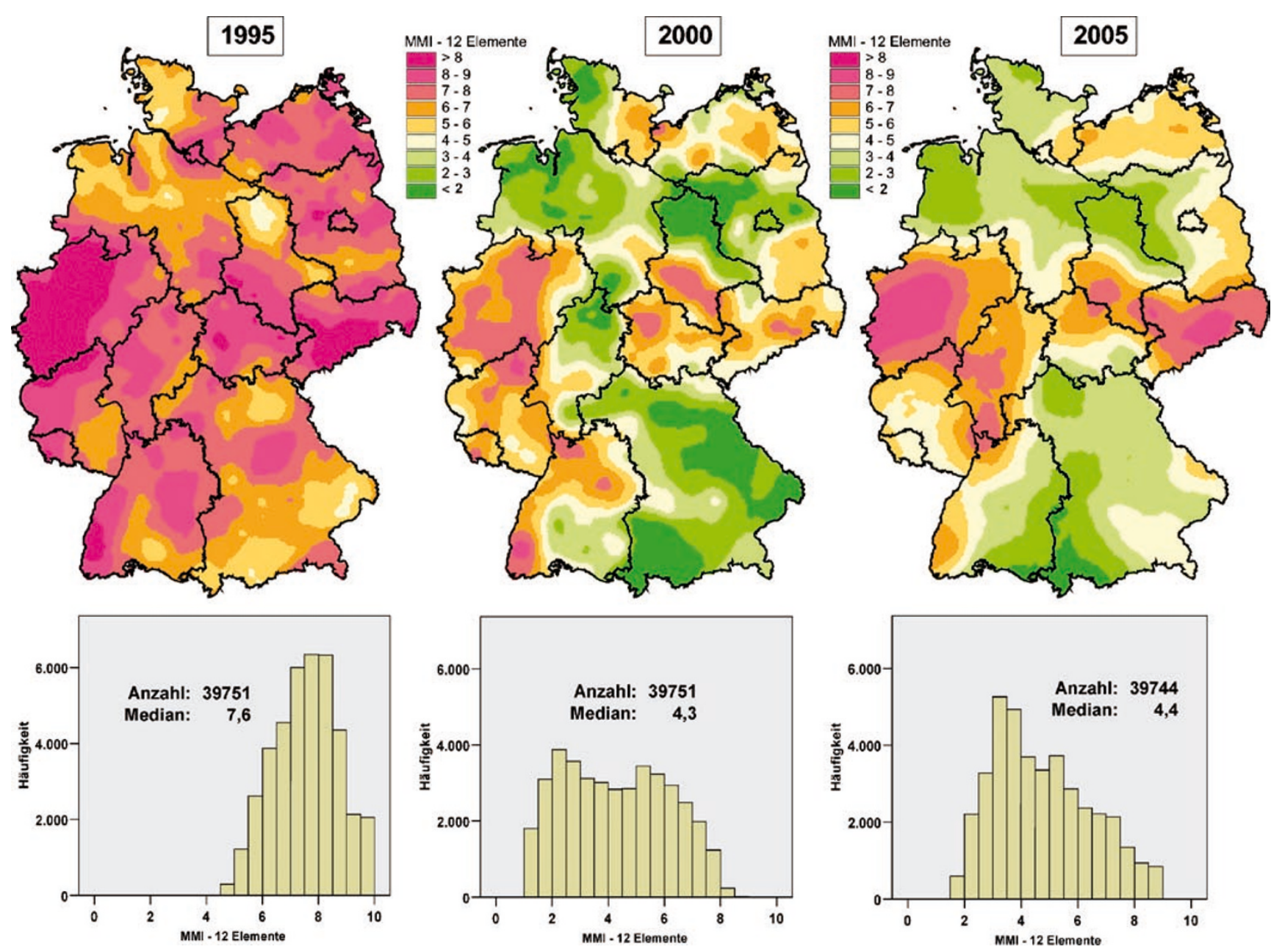

Abb. 1 Räumliche Differenzierung des MMI1990-2005

sitionsmechanismus. Hohe und sehr hohe Korrelationen bestehen zwischen den Konzentrationen von $\mathrm{As}$ und $\mathrm{Cr}$ in Moosen und in der Wet-only-Deposition. Mittlere Korrelationen weisen die Konzentrationen von $\mathrm{Cd}, \mathrm{Cu}, \mathrm{Pb}$, und $\mathrm{Zn}$ in Moosen und Depositionen auf. Die statistischen Beziehungen zwischen den Konzentrationen in Bestandsdepositionen und Moosen sind bei $\mathrm{Cd}, \mathrm{Cu}$ and $\mathrm{Pb}$ gering, mittelstark hingegen für $\mathrm{Zn}$. Die Korrelationen von $\mathrm{Cd}$ and $\mathrm{Pb}$ in Moosen und den modellierten Depositionen sind niedrig bis mittel und variieren zeitlich, stoffspezifisch und nach modelliertem Depositionsprozess: Die höchsten Korrelationen bestehen zwischen den Konzentrationen von $\mathrm{Cd}$ und $\mathrm{Pb}$ in Moosen und in der Gesamt- und Trockendeposition.

\subsubsection{Multiple Korrelationsanalyse \\ mit Entscheidungsbaumverfahren CART und CHAID}

Die ersten beiden Ebenen des CHAID-Baums für den MMI2005 sind als Dendrogramm dargestellt (Abb. 3). Demnach wurde die Entfernung der Moosentnahmestelle zur Baumkrone als statistisch signifikantester Prädiktor ausgewählt, um den Ausgangsdatensatz in drei Untergruppen aufzuteilen. Dabei werden Standorte, die in einer Entfernung von maximal $2 \mathrm{~m}$ vom Kronendach entfernt beprobt wurden, einer Klasse zugewiesen, in der im Schnitt die höchsten MMI-Werte auftauchen $(\mathrm{Mw}=6.3)$. Die anderen beiden Knoten 2 (Entfernung zur Baumkrone 2 bis $9 \mathrm{~m}$ ) und 3 (Entfernung zur Baumkrone jenseits von $9 \mathrm{~m}$ ) zeigen im Schnitt niedrigere Gehalte ( $\mathrm{Mw}=5,7$ bzw. 4,6). Die 167 Standorte, an denen Proben im unmittelbaren Traufeinfluss entnommen wurden, werden in der zweiten Ebene durch den Prädiktor „Häufigkeit des Vorkommens“ in zwei weitere Unterknoten unterteilt. Dabei zeigen diejenigen Standorte, an denen häufig Moose gefunden wurden, im Schnitt niedrigere Metallbioakkumulationen auf $(\mathrm{Mw}=5,7)$ als solche, an denen selten Moose gefunden wurden $(\mathrm{Mw}=6,9)$. Diejenigen Standorte, an denen Moose in einer Entfernung zwischen 2 und $9 \mathrm{~m}$ entnommen wurden, werden in der zweiten Ebene durch die Bewuchsform in drei Unterknoten gegliedert. Dabei zeigen diejenigen Standorte mit rasenbil- 
Tabelle 9 Bivariate Rankkorrelationskoeffizienten $(r s)$ der Metallanreicherung in Moosen

\begin{tabular}{|c|c|c|c|c|c|c|c|c|c|c|}
\hline & \multicolumn{9}{|c|}{1990} & \multirow[b]{2}{*}{$\mathrm{Zn}$} \\
\hline & As & Cd & $\mathrm{Cr}$ & $\mathbf{C u}$ & $\mathrm{Fe}$ & $\mathbf{N i}$ & $\mathbf{P b}$ & $\mathbf{T i}$ & V & \\
\hline As & 1,00 & - & - & - & - & - & - & - & - & - \\
\hline Cd & 0,65 & 1,00 & - & - & - & - & - & - & - & - \\
\hline $\mathrm{Cr}$ & 0,17 & 0,07 & 1,00 & - & - & - & - & - & - & - \\
\hline $\mathrm{Cu}$ & 0,03 & 0,02 & 0,63 & 1,00 & - & - & - & - & - & - \\
\hline $\mathrm{Fe}$ & 0,22 & 0,09 & 0,90 & 0,60 & 1,00 & - & - & - & - & - \\
\hline $\mathbf{N i}$ & 0,36 & 0,41 & 0,69 & 0,62 & 0,69 & 1,00 & - & - & - & - \\
\hline $\mathbf{P b}$ & 0,29 & 0,39 & 0,75 & 0,60 & 0,78 & 0,80 & 1,00 & - & - & - \\
\hline Ti & 0,08 & $-0,16$ & 0,82 & 0,45 & 0,84 & 0,41 & 0,55 & 1,00 & - & - \\
\hline $\mathbf{V}$ & 0,26 & 0,25 & 0,71 & 0,47 & 0,81 & 0,65 & 0,84 & 0,60 & 1,00 & - \\
\hline Zn & 0,26 & 0,47 & 0,59 & 0,52 & 0,54 & 0,73 & 0,74 & 0,27 & 0,62 & 1,00 \\
\hline
\end{tabular}

1995

\begin{tabular}{lcccccccccccc} 
& $\mathbf{A s}$ & $\mathbf{C d}$ & $\mathbf{C r}$ & $\mathbf{C u}$ & $\mathbf{F e}$ & $\mathbf{H g}$ & $\mathbf{N i}$ & $\mathbf{P b}$ & $\mathbf{S b}$ & $\mathbf{T i}$ & $\mathbf{V}$ & $\mathbf{Z n}$ \\
\hline $\mathbf{A s}$ & 1,00 & - & - & - & - & - & - & - & - & - & - & - \\
$\mathbf{C d}$ & 0,26 & 1,00 & - & - & - & - & - & - & - & - & - & - \\
$\mathbf{C r}$ & 0,26 & 0,70 & 1,00 & - & - & - & - & - & - & - & - & - \\
$\mathbf{C u}$ & 0,20 & 0,16 & 0,17 & 1,00 & - & - & - & - & - & - & - & - \\
$\mathbf{F e}$ & 0,70 & 0,39 & 0,56 & 0,26 & 1,00 & - & - & - & - & - & - & - \\
$\mathbf{H g}$ & $-0,10$ & 0,10 & 0,26 & 0,29 & 0,07 & 1,00 & - & - & - & - & - & - \\
$\mathbf{N i}$ & 0,35 & 0,70 & 0,78 & 0,16 & 0,44 & 0,30 & 1,00 & - & - & - & - & - \\
$\mathbf{P b}$ & 0,31 & 0,69 & 0,58 & 0,06 & 0,43 & 0,11 & 0,60 & 1,00 & - & - & - & - \\
$\mathbf{S b}$ & 0,10 & 0,32 & 0,36 & 0,22 & 0,21 & 0,17 & 0,32 & 0,49 & 1,00 & - & - & - \\
$\mathbf{T i}$ & 0,62 & 0,08 & 0,31 & 0,18 & 0,76 & 0,06 & 0,24 & 0,12 & 0,04 & 1,00 & - & - \\
$\mathbf{V}$ & 0,36 & 0,61 & 0,71 & 0,07 & 0,62 & 0,07 & 0,58 & 0,65 & 0,36 & 0,38 & 1,00 & - \\
$\mathbf{Z n}$ & 0,19 & 0,54 & 0,38 & 0,65 & 0,40 & 0,16 & 0,21 & 0,34 & 0,25 & 0,19 & 0,37 & 1,00 \\
\hline
\end{tabular}

2000

\begin{tabular}{lcccccccccccc} 
& $\mathbf{A s}$ & $\mathbf{C d}$ & $\mathbf{C r}$ & $\mathbf{C u}$ & $\mathbf{F e}$ & $\mathbf{H g}$ & $\mathbf{N i}$ & $\mathbf{P b}$ & $\mathbf{S b}$ & $\mathbf{T i}$ & $\mathbf{V}$ & $\mathbf{Z n}$ \\
\hline $\mathbf{A s}$ & 1,00 & - & - & - & - & - & - & - & - & - & - & - \\
$\mathbf{C d}$ & 0,57 & 1,00 & - & - & - & - & - & - & - & - & - & - \\
$\mathbf{C r}$ & 0,67 & 0,71 & 1,00 & - & - & - & - & - & - & - & - & - \\
$\mathbf{C u}$ & 0,35 & 0,38 & 0,39 & 1,00 & - & - & - & - & - & - & - & - \\
$\mathbf{F e}$ & 0,82 & 0,56 & 0,86 & 0,40 & 1,00 & - & - & - & - & - & - & - \\
$\mathbf{H g}$ & 0,76 & 0,71 & 0,70 & 0,59 & 0,75 & 1,00 & - & - & - & - & - & - \\
$\mathbf{N i}$ & 0,53 & 0,66 & 0,76 & 0,41 & 0,69 & 0,72 & 1,00 & - & - & - & - & - \\
$\mathbf{P b}$ & 0,68 & 0,87 & 0,76 & 0,40 & 0,67 & 0,78 & 0,69 & 1,00 & - & - & - & - \\
$\mathbf{S b}$ & 0,34 & 0,56 & 0,58 & 0,49 & 0,39 & 0,43 & 0,52 & 0,65 & 1,00 & - & - & - \\
$\mathbf{T i}$ & 0,69 & 0,39 & 0,59 & 0,32 & 0,73 & 0,57 & 0,50 & 0,47 & 0,25 & 1,00 & - & - \\
$\mathbf{V}$ & 0,63 & 0,70 & 0,81 & 0,35 & 0,79 & 0,71 & 0,70 & 0,77 & 0,54 & 0,48 & 1,00 & - \\
$\mathbf{Z n}$ & 0,56 & 0,78 & 0,56 & 0,48 & 0,49 & 0,57 & 0,38 & 0,71 & 0,50 & 0,36 & 0,56 & 1,00 \\
\hline
\end{tabular}

2005

\begin{tabular}{lcccccccccccc} 
& $\mathbf{A s}$ & $\mathbf{C d}$ & $\mathbf{C r}$ & $\mathbf{C u}$ & $\mathbf{F e}$ & $\mathbf{H g}$ & $\mathbf{N i}$ & $\mathbf{P b}$ & $\mathbf{S b}$ & $\mathbf{T i}$ & $\mathbf{V}$ & $\mathbf{Z n}$ \\
\hline $\mathbf{A s}$ & 1,00 & - & - & - & - & - & - & - & - & - & - & - \\
$\mathbf{C d}$ & 0,60 & 1,00 & - & - & - & - & - & - & - & - & - & - \\
$\mathbf{C r}$ & 0,53 & 0,26 & 1,00 & - & - & - & - & - & - & - & - & - \\
$\mathbf{C u}$ & 0,48 & 0,50 & 0,42 & 1,00 & - & - & - & - & - & - & - & - \\
$\mathbf{F e}$ & 0,82 & 0,67 & 0,60 & 0,57 & 1,00 & - & - & - & - & - & - & - \\
$\mathbf{H g}$ & 0,74 & 0,64 & 0,39 & 0,63 & 0,76 & 1,00 & - & - & - & - & - & - \\
$\mathbf{N i}$ & 0,47 & 0,58 & 0,17 & 0,39 & 0,68 & 0,68 & 1,00 & - & - & - & - & - \\
$\mathbf{P b}$ & 0,66 & 0,91 & 0,19 & 0,43 & 0,69 & 0,68 & 0,65 & 1,00 & - & - & - & - \\
$\mathbf{S b}$ & 0,26 & 0,49 & $-0,04$ & 0,38 & 0,37 & 0,47 & 0,58 & 0,61 & 1,00 & - & - & - \\
$\mathbf{T i}$ & 0,68 & 0,47 & 0,49 & 0,53 & 0,78 & 0,58 & 0,48 & 0,47 & 0,28 & 1,00 & - & - \\
$\mathbf{V}$ & 0,57 & 0,75 & 0,48 & 0,49 & 0,72 & 0,68 & 0,58 & 0,79 & 0,50 & 0,49 & 1,00 & - \\
$\mathbf{Z n}$ & 0,36 & 0,63 & 0,51 & 0,70 & 0,46 & 0,37 & 0,22 & 0,45 & 0,14 & 0,40 & 0,54 & 1,00 \\
\hline
\end{tabular}


Tabelle 10 Bivariate Rankkorrelationskoeffizienten $(r s)$ zwischen Metallkonzentrationen in Moosen und in Depositionen

\section{ICP Forests Level II}

Bulk Deposition (Freiland)

Bulk Deposition (Kronentraufe)

\begin{tabular}{|c|c|c|c|c|c|c|c|c|c|c|c|c|c|c|c|c|}
\hline \multirow[t]{2}{*}{ Element } & \multicolumn{2}{|c|}{1998} & \multicolumn{2}{|c|}{1999} & \multicolumn{2}{|c|}{2000} & \multicolumn{2}{|c|}{$\Sigma 98-00$} & \multicolumn{2}{|c|}{1998} & \multicolumn{2}{|c|}{1999} & \multicolumn{2}{|c|}{2000} & \multicolumn{2}{|c|}{$\Sigma 98-00$} \\
\hline & $\mathbf{n}$ & rs & n & rs & $\mathbf{n}$ & rs & $\mathbf{n}$ & rs & $\mathbf{n}$ & rs & n & rs & $\mathbf{n}$ & rs & n & rs \\
\hline $\mathrm{Cd}$ & 18 & 0,5 & 18 & 0,3 & 18 & $-0,1$ & 18 & 0,2 & 20 & 0,2 & 20 & 0,1 & 20 & 0,0 & 20 & 0,0 \\
\hline $\mathrm{Cu}$ & 17 & 0,4 & 17 & 0,5 & 17 & 0,0 & 17 & 0,4 & 17 & 0,3 & 17 & 0,3 & 17 & $-0,2$ & 17 & 0,3 \\
\hline $\mathrm{Pb}$ & 19 & 0,3 & 19 & 0,5 & 19 & 0,5 & 19 & 0,5 & 21 & 0,0 & 21 & 0,0 & 21 & 0,1 & 21 & 0,0 \\
\hline $\mathrm{Zn}$ & 27 & 0,5 & 27 & 0,5 & 27 & 0,5 & 27 & 0,5 & 32 & 0,6 & 32 & 0,6 & 32 & 0,3 & 32 & 0,6 \\
\hline
\end{tabular}

UBA/ICP Integrated Monitoring

Wet only (Freiland)

\begin{tabular}{lcccccc} 
Element & $\mathbf{2 0 0 4}$ & & $\mathbf{2 0 0 5}$ & \multicolumn{3}{c}{$\mathbf{\Sigma 0 4 - 0 5}$} \\
\hline $\mathrm{As}$ & 6 & 0,6 & 6 & 0,6 & 6 & 0,7 \\
$\mathrm{Cd}$ & 6 & $\mathbf{0 , 9}$ & 6 & $\mathbf{1 , 0}$ & 6 & $\mathbf{1 , 0}$ \\
$\mathrm{Cr}$ & 6 & 0,3 & 6 & 0,0 & 6 & 0,3 \\
$\mathrm{Zn}$ & 6 & 0,0 & 6 & 0,4 & 6 & 0,1 \\
\hline
\end{tabular}

Fett: $\mathrm{p}<0,10$

Fett kursiv: $\mathrm{p}<0,05$
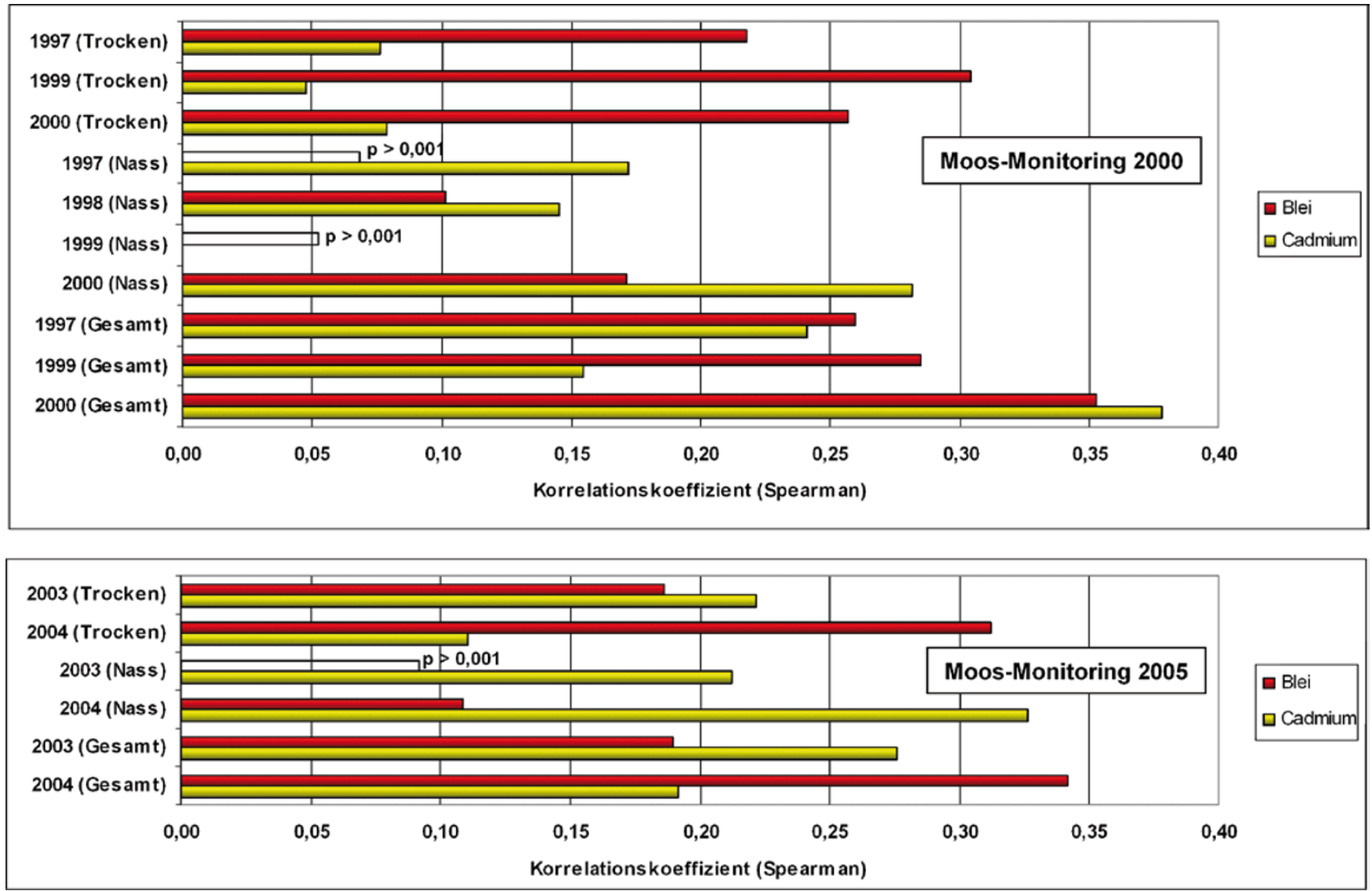

Abb. 2 Korrelationen $(\mathrm{p}<0.001)$ für $\mathrm{Cd}, \mathrm{Pb}$; moosmodellierte Deposition (seminatürliche Vegetation)

dendem Moosbewuchs höhere MMI-Werte auf als solche mit polsterartigem Bewuchs $(\mathrm{Mw}=4,8$ im Knoten 6 gegenüber $\mathrm{Mw}=5,8 \mathrm{im}$ Knoten 7). Letztere wiederum zeigen im Schnitt niedrigere Werte auf als solche, an denen spärlicher Bewuchs vorherrschte ( $\mathrm{Mw}=5,8$ im Knoten 7 gegenüber $\mathrm{Mw}=6,8 \mathrm{im}$ Knoten 8). Diejenigen 192 Standorte, 


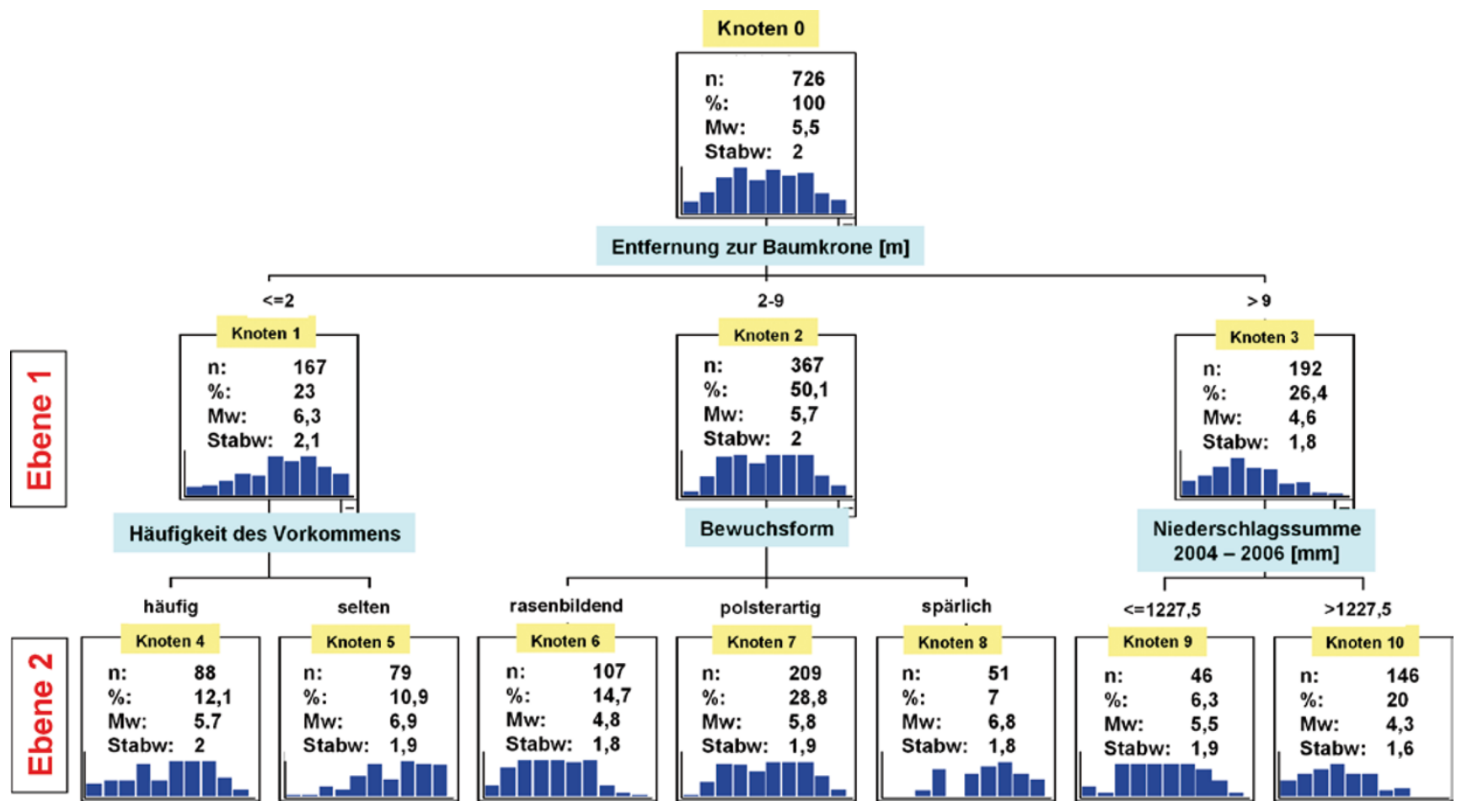

Abb. 3 CHAID-Dendrogramm für den $\mathrm{MMI}_{2005}$ (Ebene 1 und 2)

die jenseits von $9 \mathrm{~m}$ von der nächsten Baumkrone entfernt lagen, werden durch die Niederschlagssumme innerhalb des zweijährigen Akkumulationszeitraums in zwei weitere Unterklassen unterteilt. Hierbei zeigen Standorte mit hohen Niederschlagssummen niedrigere Metallbioakkumulationen ( $\mathrm{Mw}=4,3$ in Knoten 10 gegenüber $\mathrm{Mw}=5,5$ in Knoten 9). Aus dem weiteren, hier nicht dargestellten Verlauf des Dendrogramms lässt sich entnehmen, dass der Prädiktor „Häufigkeit des Vorkommens“ drei weitere Male als statistisch signifikantester Prädiktor ausgewählt wurde. Es zeigen sich dieselben Tendenzen wie oben beschrieben: Niedrigere Metallbioakkumulationen bei Standorten mit häufigem Vorkommen der jeweiligen Moosart sowie hohe Metallbioakkumulationen bei Standorten mit seltenem Vorkommen.

In einem entsprechenden zur Kontrolle berechneten CART-Baum erfolgt die Unterteilung des Ausgangsdatensatzes auch anhand der Entfernung des Probenentnahmestandorts zur Baumkrone: Der Unterknoten 1 mit 473 Standorten, die allesamt in einer Entfernung von höchstens 7,5 m beprobt wurden, zeigt dabei im Schnitt höhere MMI-Werte auf als der Unterknoten 2, in dem 253 Standorte eingruppiert wurden $(\mathrm{Mw}=5,9$ gegenüber $\mathrm{Mw}=4,7)$. Standorte mit Entfernungen unter 7,5 m zur nächsten Baumkrone werden anhand des Prädiktors „Häufigkeit des Vorkommens“ weiter untergliedert. Bei häufigem Moosvorkommen liegt der MMI im Schnitt bei 5,4 und bei seltenem Vorkommen bei 6,7. Standorte mit Entfernungen von mehr als 7,5 m zur nächsten Baumkrone werden gemäß der Niederschlagssum- men im Akkumulationszeitraum (2003-2004) weiter unterteilt. Wie beim CHAID-Baum zeigen Standorte mit hohen Niederschlagssummen auch hier niedrigere Metallbioakkumulationen $(\mathrm{Mw}=5,4$ in Knoten 5 gegenüber $\mathrm{Mw}=4,3$ in Knoten 6). Im weiteren Verlauf des CART-Baums werden in den Ebenen 3 bis 7 in vier Fällen Prädiktoren gewählt, die mit urbanen oder verkehrsbedingten Emissionen in Verbindung zu bringen sind. In zwei Fällen erfolgt die Unterteilung der Unterknoten anhand der Bestandeshöhe und des Ausgangsgesteins. Einmal ausgewählt werden die Moosart, die Landnutzung, die Niederschlagssummen im Akkumulationszeitraum (2004-2006) sowie die Höhe über NN.

\section{Diskussion}

Die Diskussion der am Beispiel des deutschen Beitrags zum Moosmonitoring dargestellten Ergebnisse wird vorwiegend in Bezug auf die entsprechenden europaweiten Resultate geführt, die von von Vertretern einiger Teilnehmerstaaten vertiefend behandelt und von Harmens et al. (2008) zusammengefasst wurden.

\subsection{Moosproben-Sammlung}

In einem ökologisch so differenzierten Raum wie Deutschland ist es nicht möglich, in einem flächendeckenden Netz seit 1995 weitgehend ortstreuer Moosentnahmestandorte 
nur eine Moosart zu sammeln. Insofern muss auf mehrere Moosarten zurückgegriffen werden. Nach der Erweiterung des Messnetzes um das Gebiet der ehemaligen DDR blieb der Anteil der prioritären Moosart Pleurozium schreberi bei $42 \%$ bis $45 \%$. Im europaweiten Messnetz lag der Anteil dieser Moosart 2005 bei $41 \%$. Im deutschen Messnetz folgen Scleropodium purum (Anteil seit 1995 zwischen $28 \%$ und $33 \%$ ), Hypnum cupressiforme (Anteil seit 1995 zwischen $16 \%$ und $25 \%$ ) sowie Hylocomium splendens (Anteil seit 1995 zwischen 0,6\% und 6,8\%). Im europäischen Messnetz folgen im Jahr 2005 auf Pleurozium schreberi (41\%) Hylocomium splendens (23\%, Hypnum cupressiforme (18\%), Sceropodium purum (12\%) und andere Arten (7\%) (Harmens et al. 2008). Wie Siewers et al. (2000) kommen Harmens et al. (2008) zu dem Ergebnis, dass die Metallakkumulation je nach Metall und Region artenspezifisch sein kann. Folgende Tendenzen zeigten sich im europaweiten Datensatz 2005 ( $\mathrm{Hc}=$ Hypnum cupressiforme, Hs = Hylocomium splendens, Ps $=$ Pleurozium schreberi, rectus: durchschnittliche Differenz 10-20\%, fett durchschnittliche Differenz $>20 \%$ ): 1 . Hs > Ps bei As, $\mathrm{Cr}, \mathrm{Cu}, \mathrm{Fe}, \mathrm{Hg}, \mathrm{Ni}$, $\mathrm{V}, \mathrm{Al}, \mathrm{Sb}, 2 . \mathrm{Hc}>\mathrm{Ps}$ bei As, $\mathrm{Cd}, \mathrm{Cr}, \mathrm{Fe}, \mathrm{Hg}, \mathrm{Ni}, \mathrm{Pb}, \mathrm{Zn}, 3$. $\mathrm{Hc}<\mathrm{Ps}$ bei $\mathrm{Cu}, \mathrm{Al}, \mathrm{Sb}, 4 \mathrm{Hs}>\mathrm{Hc}$ bei As, $\mathrm{Cr}, \mathrm{Cu}, \mathrm{Hg}, \mathrm{V}$ und 5. Hs $<\mathrm{Hc}$ bei Ni. Eine europaweite oder länderspezifische Umrechnung der Metallkonzentrationen auf eine Art würde jedoch zusätzliche Unschärfen erzeugen - insbesondere dann, wenn die Konzentrationsbereiche der Metalle regional variieren. Hinzu kommt, dass das statistische Design des Moosmonitorings es nicht ermöglicht, die mit den verschiedenen Moosarten statistisch assoziierten Metallgehalte zwingend so zu interpretieren, dass die Unterschiede alleine durch eine unterschiedliche Sammeleffizienz bedingt ist. Eine solche Interpretation wäre nur dann zulässig, wenn die miteinander verglichenen Metallgehalte von Moosarten stammen, die an jeweils einem Standort gesammelt wurden. Dies ist jedoch nicht der Fall. Geeigneter als Umrechnungsfaktoren ist es deshalb, die statistische Verknüpfung der Moosart mit der Varianz der Metallkonzentrationen räumlich differenzierend zu analysieren, wie es in dieser Untersuchung mit Entscheidungsbaumverfahren erfolgt ist (Abschn. 2.6.2., 3.6.2).

\subsection{Qualitätskontrolle}

Die anhand von QC/QA-Prozeduren und Plausibilitätserwägungen in allen Teilnehmerstaaten überprüften Daten wurden durch das ICP-Programmzentrum nochmals anhand der Anforderungen von Steinnes et al. (1997) in Bezug auf die Referenzmaterialien M2 und M3 sowie anhand der Ergebnisse des Ländergrenzen überschreitenden Austauschs von Probenmaterial analysiert und bewertet. Smodiš und Bleise (2007) ergänzten die Überprüfung der Qualitätskontrollkenndaten durch eine eigenständige Qualitätskontrollstudie, in der die Referenzmaterialien M2 und M3 von 17 Laboratorien aus 15 Staaten untersucht wurden. Insgesamt konnten die integrative Analyse und Bewertung der einschlägigen QC/QA-Kenndaten belegen, dass die Qualitätskriterien Richtigkeit, Präzision und Vergleichbarkeit der Daten nicht nur in Deutschland, sondern auch über die Teilnehmerstaaten und Messkampagnen hinweg eingehalten wurden. Die Ergebnisse der grenzüberschreitenden Qualitätskontrolle zwischen Deutschland und seinen unmittelbar angrenzenden Nachbarstaaten sind zufriedenstellend und geben keine Hinweise darauf, dass methodenbedingte Messwertesprünge an den Staatengrenzen vorliegen. Deshalb können die räumlichen und zeitlichen Trends als solche interpretiert werden und sind nicht auf systematische Fehler zurückzuführen. Dies wird auch durch die Korrelationen der Metallgehalte in Moosen mit Informationen aus Emissionskatastern und Depositionsmessnetzen - für Deutschland (Abschn. 3.6) wie für Europa insgesamt - bestätigt (Abschn. 4.6).

Die Qualitätskontrolle aller Messdaten und Qualitätskontrollkenndaten umfasste auch die Analyse und Bewertung der mit verschiedenen Moosarten statistisch verknüpften Messwertvarianz (Absch. 4.1, 2.6.2, 3.6.2), der standortinternen Variabilität sowie der mit der Jahreszeit der Probenentnahme assoziierten Messwertunterschiede, der Varianz innerhalb der Mischproben sowie der mit der Moospräparation, Aufschlusstechnik und Analysenmethode verknüpften Varianz. Faktoren, die mit Messwertunterschieden korrelieren, wurden identifiziert und ergaben diese Rangfolge: Jahreszeit der Probenentnahme (regional spezifisch) > Moosart (regional und Metall spezifisch) $=$ standortinterne Varianz $>$ analytische Technik (Harmens et al. 2008).

\subsection{Plausibilitätskontrolle}

Die zusätzlich zur Qualitätskontrolle der analytischen Daten durchgeführte Plausibilitätsprüfung führte in Deutschland nur vereinzelt zum Ausschluss von Messdaten aus den Auswertungen (Abschn. 3.3). Im Vergleich zur Qualitätskontrolle ist die Plausibilitätsprüfung stärker von Experteneinschätzungen geprägt. In Ergänzung zur Überprüfung der Qualitätskontrollkenndaten aus den Teilnehmerstaaten erfolgte auch eine Plausibilitätsprüfung durch das Programmzentrum ICP Vegetation. Ergaben sich hierbei Auffälligkeiten, wurden solche Befunde dem jeweiligen Teilnehmerstaat mit der Aufforderung zur Klärung übermittelt. Gelang eine Klärung nicht, so wurden solche Befunde aus dem europäischen Datensatz und von der Kartierung ausgeschlossen (Harmens et al. 2008).

\subsection{Geostatistik}

Die Zielwerte des Nugget/Sill-Verhältnisses und der Kreuzvalidierungskennwerte (Abschn. 2.4) werden wie auch in 
vielen anderen geostatistischen Analysen von Umweltdaten nicht hundertprozentig erreicht (Auerswald et al. 2009; Kerry und Oliver 2008; Le Corre et al. 1998). Betrachtet man das Nugget/Sill-Verhältnis (N/S) als Indikator für die räumliche Abhängigkeit der Messwerte (Cambardella et al. 1994) und klassifiziert diese nach Zhao und Cai (2004) als schwach, wenn N/S $>0,75$, als mittel, wenn N/S Werte zwischen 0,75 und 0,25 annimmt, sowie als stark, wenn $\mathrm{N} / \mathrm{S}<0,25$, dann sind die räumlichen Autokorrelationen der Metall-Bioakkumulation 2005 in Deutschland überwiegend der mittleren Ausprägungsstufe zuzuordnen. Die Qualität der Flächenschätzung sollte letztlich daran beurteilt werden, ob sie im Rahmen der, anhand von Mehrfachbeprobungen an jeweils einem von hinreichend vielen Standorten empirisch bestimmten, Standort-Varianz liegt oder nicht. Die Mediane der prozentualen Schätzfehler (MPE) liegen voll in dem Bereich der von Siewers et al. (2000) an jeweils einem von 49 Moossammelorten bestimmten empirischen Varianz einer breiten Palette von Metallen. Das bedeutet, dass die (Un)schärfe der räumlichen Schätzung nicht größer ist als die (Un)schärfe der räumlich punktuellen Messung. Mehr kann man von Schätzverfahren nicht erwarten.

\subsection{Zeitliche Entwicklung}

der elementspezifischen Bioakkumulation

und der Multi-Metall-Indizes (MMI)

Das Moosmonitoring belegt, dass abnehmende MetallEmissionen und -Depositionen in Deutschland zu sinkenden Metall-Konzentrationen in Moosen führen. Doch im Monitoring 2005 wurden bei einigen Metallen gegenüber 2000 statistisch signifikant erhöhte Werte nachgewiesen, die insbesondere bei $\mathrm{Cr}$ deutlich waren. Diese Trends stimmen mit denen in anderen europäischen Staaten überein: Zwischen 1990 und 2005 sanken die Konzentrationen am deutlichsten im Falle von $\mathrm{Pb}(72,3 \%)$, As $(71,8 \%)$, V (60,4\%), Cd $(52,2 \%)$ und $\mathrm{Fe}(45,2 \%)$. Moderate Abnahmen wurden für $\mathrm{Zn}(29,3 \%), \mathrm{Cu}(20,4 \%)$ und $\mathrm{Ni}(20,0 \%)$ ermittelt. Geringe Reduktionen wurden hingegen für $\mathrm{Cr}$ nachgewiesen (2\%). Diese Tendenzen stimmen gut überein mit den EMEP-Emissionsdaten bzw. den modellierten Depositionen von As, Cd, $\mathrm{Cu}, \mathrm{Pb}, \mathrm{Hg}$ und $\mathrm{Zn}$ (Harmens et al. 2008). Dies trifft auch für $\mathrm{Ni} \mathrm{zu}$, obwohl die Abnahmen der Ni-Konzentrationen in Moosen geringer ausfallen als die der Emissionen. $\mathrm{Cr}$ folgt dieser Entwicklung nicht: Obwohl die Cr-Emissionen abnehmen, gilt dies kaum für die Cr-Konzentrationen in Moosen. Diese zweiteilige zeitliche Entwicklung 1990 bis 2000 und 2000 bis 2005 gibt der MMI zutreffend Metalle übergreifend und räumlich differenziert wieder. Am Beispiel des deutschen Moosmonitorings wird klar, dass europaweite Trends der Metall-Akkumulation regional und lokal stoffspezifisch variieren.
4.6 Zusammenhänge der Bioakkumulation

mit Deposition sowie Eigenschaften

der Moossammelorte und ihrer Umgebung

\subsubsection{Bivariate Korrelationsanalyse}

Atmosphärische Deposition ist der Stofffluss aus der Atmosphäre auf die Erdoberfläche: Gase sowie sedimentierende und nicht sedimentierende Tropfen und Partikel werden aus der Atmosphäre aufOberflächen (Akzeptoren) biotischer oder abiotischer Systeme über und reichern sich dort an. Biotische Akzeptoren sind die oberirdischen Sprossteile von Pflanzen, insbesondere die Blätter und Nadeln. Abiotische Akzeptoren können der Boden, Oberflächengewässer, Schneedecken oder Bauwerke sein. Die atmosphärische Deposition ist ein aus vielen Einzelmechanismen bestehender Vorgang: Größere Partikel und Tropfen folgen der Schwerkraft und sedimentieren. Schwebende Tropfen und Partikel werden mit Luftströmungen auf Oberflächen abgelagert (Impaktion). Gase werden an feuchten Oberflächen gelöst oder von trockenen Oberflächen adsorbiert. Trockene Deposition ist der Austrag sedimentierender Partikel sowie von Gasen aus der Atmosphäre einschließlich ihrer Ablage auf Akzeptoroberflächen. Austrag und Ablage von Stoffen, die in Regen, Schnee und Hagel inkorporiert sind, wird als nasse atmosphärische Deposition bezeichnet. Mit der nassen Deposition werden gelöste und ungelöste Schadstoffe durch die Niederschläge wie z.B. Regen, Schnee oder Graupel aus der Atmosphäre entfernt. Dies kann innerhalb von Wolken (Rainout) oder unterhalb der Wolkenbasis (Washout) erfolgen. Die Effektivität dieses Vorgangs hängt ab von den Eigenschaften der ausgewaschenen Substanzen (Löslichkeit, Partikelgröße) und den Niederschlagscharakteristika (Niederschlagsintensität, Tropfenspektrum). Die Wolken können die gebundenen Luftverunreinigungen aus verschiedenen Emissionsquellen zu entfernteren Gebieten transportieren. Dabei verändert sich das Verhältnis von trockener zu nasser Deposition von 10:1 in Emittentennähe auf 1:1 in emittentenfernen Gebieten. Die nasse Deposition nimmt mit der Entfernung zur Emissionsquelle gegenüber der trockenen Deposition zu. Der Austrag von Stoffen aus der Atmosphäre durch Deposition ist kein vollständig irreversibler Prozess. Ein Teil der deponierten Stoffmenge kann wieder zurück in die Atmosphäre verdampfen (Re-Emission). Oberirdische Pflanzenteile, insbesondere Blätter und Nadeln in Wäldern, stellen eine sehr effektive Akzeptoroberfläche dar (Auskämmeffekt). Bei Niederschlagsereignissen können die zuvor trocken deponierten und ggf. zwischenzeitlich z.T. metabolisierten Stoffe abgewaschen werden und über die Kronentraufe und den Stammablauf auf den Boden und dort wachsende Moose gelangen (Interzeptionsdeposition). Von der Vegetation ausgekämmte Nebel-/Wolkentröpfchen enthalten gelöste Stoffe, deren Eintrag als okkulte Deposition bezeichnet wird. Durch Interzeptionsdeposition und okkulte 
Deposition ist der Stoffeintrag in Wäldern, insbesondere in den nicht-winterkahlen Nadelwäldern, gegenüber der Deposition auf unbewaldeten Standorten (,Freiland“) erhöht, und die in den Moosen angereicherten Stoffe sind das Ergebnis von Nass-, Trocken- und Interzeptionsdeposition sowie der okkulten Deposition (Guderian und Gunkel 2001).

Die korrelationsstatistische Analyse zeigt, dass die Anreicherung atmosphärischer Depositionen nicht nur räumlich variiert (Abschn. 4.5), sondern auch zeitlich (Tabelle 10). Aus den zeitlich variablen Beträgen der Korrelationen zwischen den in den Moosen akkumulierten Metallen lässt sich ableiten, dass auch die Ausprägung der statistischen Beziehungen zwischen den Metall-Konzentrationen in den Moosen und in den Depositionen nicht immer und überall hoch ausgeprägt erwartet werden kann. Deposition und Bioakkumulation sind miteinander verknüpfte Vorgänge, sodass Korrelationen plausibel sind. Die biologische Anreicherung von Metallen ist dabei v. a. ein biochemischer und kein rein physikalischer Prozess. So werden beim Moosmonitoring zwar auch auf der Moosoberfläche adsorptiv gebundene Partikel chemisch-analytisch erfasst. Metalle können sich aber auch in den Moosen direkt anreichern. Herpin (1997) unterscheidet diesbezüglich die passive, extrazelluläre sowie die aktive, intrazelluläre Aufnahme. Die aktive intrazelluläre Aufnahme ist dabei ein energieaufwendiger Prozess und erfolgt über die Lipoproteine der Zellmembran. Die passive Aufnahme von Metallionen geschieht über Ionenaustauschvorgänge pektinhaltiger Substanzen, die an den Zellwänden lokalisiert sind. Die negativ geladenen, an Austauscherplätze gebundenen Kationen (z.B. Ca2+ oder $\mathrm{Mg} 2+$ ) werden dabei durch z. B. Schwermetallkationen ersetzt, die eine höhere Affinität zu diesen Austauschergruppen besitzen. Gleichwohl ist, abgesehen von der raumzeitlichen Varianz, nicht davon auszugehen, dass der Betrag der Korrelation zwischen Deposition und Bioakkumulation 1 ist, da beide Prozesse nicht identisch sind, sondern zwei aufeinander folgende Glieder einer Prozesskette (Lindberg und Turner 1988), die räumlich und zeitlich so variabel sind, dass die in sie eingebundenen Umweltkompartimente Stoffkonzentrationen aufweisen, die nicht immer plausibel erscheinen (Fränzle und Schimming 2008). So fehlt beispielsweise im Acadia National Park (Mount Desert Island, Maine) jegliche punktförmige Hg-Quelle, doch werden dort die höchsten Bio-Akkumulationen von $\mathrm{Hg}$ in den USA nachgewiesen (Diehl und Beard 2009). Zu dieser negativen Korrelation gibt es langjährige Forschungen über den Hg-Transfer und darauf aufbauende Theorien über die $\mathrm{Hg}$ Transfers zwischen anorganischen und organischen Vorräten sowie über biologische Anreicherung bzw. Biomagnifikation entlang trophischer Ebenen. Es wurde herausgefunden, dass die Insellage des Acadia National Park eine natürliche biogeochemische Grenze für nicht-atmosphärische Hg-Vorräte bildet. Diese Erkenntnis wurde durch Integration und Georeferenzierung von Daten sehr unterschiedlicher Phänomenbe- reiche wie beispielsweise Landschaftselemente und abiotische Kenngrößen in einem GIS ermöglicht (Diehl und Beard 2009). Auf diese Art konnte geklärt werden, in welcher Weise die komplizierten Beziehungen zwischen Stoffquellen und -senken durch meteorologische Bedingungen sowie die Eigenschaften der Messorte und ihrer Umgebung räumlich variiert werden können. Von den standörtlichen und regionalen Landschaftsmerkmalen erwiesen sich insbesondere die horizontale und vertikale Struktur der Vegetation, die Landnutzungsmuster sowie die Bodeneigenschaften als bedeutsam für Deposition und Akkumulation.

Neben diesen standörtlichen und regionalen Landschaftseigenschaften wird die Bioakkumulation auch durch die Fähigkeit der Moose gesteuert, ihren Stoffhaushalt auf die stofflichen Randbedingungen am Wuchsort einzustellen. Hierbei interagieren die deponierten Metalle physikalisch durch Adsorption und biochemisch, so z. B. ligandenchemisch durch Komplexbildung. Die Biokonzentration von Metallen hängt also von ihren Stoffeigenschaften, ihrer Verteilung in den Vegetationsschichten und der biochemischen Beschaffenheit der biologischen Rezeptoren ab (Fränzle und Schimming 2008). Bezogen auf die im Moosmonitoring 2005 gegenüber den vorherigen Messkampagnen erhöhten Cr-Werte könnte dies Folgendes bedeuten: $\mathrm{Cr}$ ist im Boden mobil, falls es als Chromat(VI) vorliegt. Dieses wird durch katalytische Oxidation an Manganoxiden aus Cr(III) und Luftsauerstoff gebildet. Chromat(VI) reagiert im O- und A-Horizont mit Phenolen sowie mit Aminosäuren unter Oxidation und nachfolgender Bildung der dann für Pflanzen resorbierbaren Cr(III)-Aminosäure- oder Phenolcarboxylatkomplexe. Moose geben unter Trockenstress Aminosäuren in den Boden ab. Wenn also Trockenstress an einer Moosdecke einsetzt, wächst die Bioverfügbarkeit von $\mathrm{Cr}$ für die dort vorkommenden Pflanzen einschließlich der Moose selbst, die bei Trockenheit und spärlicher Bodenbedeckung Metalle aus dem Boden durch Staubeintrag aufnehmen können (Fränzle 2007; Fränzle und Schimming 2008). Diese durch Trockenstress induzierte Prozesskette hätte nichts mit einer im Untersuchungszeitraum steigenden $\mathrm{Cr}$-Deposition zu tun, sondern mit biogeochemischen Interaktionen zwischen atmosphärischem Zustand (Trockenheit, Luftbewegung), Reaktion der Moosdecke, Bodenbedeckung und -eigenschaften (Erodierbarkeit durch Wind). Die Erklärungskraft dieser Hypothese ließe sich dadurch prüfen, ob sich diese Zusammenhänge auch lokal und regional bei anderen Pflanzen nachweisen lassen und ob deren Veränderungen mit der Anwesenheit oder Zunahme von Trockenstress exponierten Moospolstern korrelieren. Alternativ wäre zu klären, ob die Emission oder Deposition von Cr zugenommen hat. Dies scheiterte in dieser Untersuchung an der Unzugänglichkeit der entsprechenden - vorhandenen - Daten. Die Verknüpfung von Daten, die aneinander gekoppelte Prozesse abbilden, ist unabdingbar für die Erklärung von Phänomenen wie der Stoffanreicherung in Ökosystemen. 
Sieht man davon ab, dass die nicht nur in Deutschland, sondern u. a. auch in der Schweiz erhöhten Cr-Werte von Moosen nicht geklärt werden konnten, so bleibt festzuhalten, dass die Metallkonzentrationen in den Moosen mit den räumlich sehr lückenhaft und nur für wenige Metalle verfügbaren Depositionsdaten zeitlich variabel und metallspezifisch korreliert sind. Für Europa zeigt sich, dass die Metallkonzentrationen in Moosen auf zwei räumlichen Ebenen mit Begleitfaktoren korrelieren: kleinräumig mit Eigenschaften der Moossammelorte und regional mit Merkmalen in ihrer Umgebung bis zu mindestens $100 \mathrm{~km}$. Bei den regionalen Einflüssen auf die Metallkonzentrationen weisen die $\mathrm{Cd}$ - und $\mathrm{Pb}$-Konzentrationen in den Moosen die engsten statistisch signifikanten Beziehungen $(\mathrm{p} \leq 0.01) \mathrm{zu}$ den entsprechenden Emissionsraten (EMEP) auf ( $r s=0.40$ bzw. $r s=0.45)$ und zum Anteil urbaner Flächen im Umkreis vom $100 \mathrm{~km}(r s=0.43$ bzw. $r s=0.44)$ auf. Die Konzentrationen von $\mathrm{Cd}$ und $\mathrm{Pb}$ in Moosen sind auch untereinander korreliert $(r s=0.65, \mathrm{p} \leq 0.01)$, und für beide Metalle lassen sich europaweit räumliche Autokorrelationen geostatistisch nachweisen. Hg hingegen zeigt weder klare räumliche Strukturen noch nennenswerte statistische Beziehungen zu Emissions- oder Landnutzungsmustern (Holy et al. 2009a).

Ferner ist belegt, dass die Konzentrationen von $\mathrm{Cd}, \mathrm{Hg}$ und $\mathrm{Pb}$ in Moosen europaweit mit den Daten der modellierten Gesamtdeposition (EMEP) korreliert sind. Hierbei variieren die Beträge der statistisch signifikanten Korrelationen großräumlich: Die höchsten Korrelationen finden sich in Regionen mit einem hohen Anteil nasser Deposition am Gesamteintrag, die niedrigsten bei hohem Anteil trockener Deposition in Südeuropa. Dass die Korrelationen zum Süden hin abnehmen, liegt auch daran, dass die empirischen Daten, die in die Modellierung einfließen, die nicht-sedimentierende trockene Deposition nicht abbilden (Ilyin 2009).

\subsubsection{Multiple Korrelationsanalyse mit Entscheidungsbaumverfahren CART und CHAID}

Die geostatistische Analyse belegt für das Gebiet der Bundesrepublik Deutschland wie für Europa, dass die Metallkonzentrationen in den Moosen auf zwei räumlichen Ebenen variieren: kleinräumig, beeinflusst von den Eigenschaften der Moossammelorte und großräumig (Abschn. 3.4, 4.4). Welche Einflussfaktoren dabei mit den Stoffgehalten in den Moosen statistisch verknüpft sind, ließ sich in einem ersten Schritt durch bivariate Korrelationsanalyse klären (Abschn. 2.6.1, 3.6.1). In einem zweiten Schritt wurden mehrfaktorielle Einflüsse untersucht und die Relevanz der einzelnen Faktoren hierarchisiert (Abschn. 3.6.2, 3.6.2). Für das Gebiet Deutschlands wurden entsprechende Untersuchungen anhand der Daten aus dem Moosmonitoring 2000 bereits von Kleppin et al. (2008a), Pesch und Schröder (2006) sowie Schröder und Pesch (2005) vorgestellt, für Frankreich von Holy et al. (2009b), für mehrere europäische Länder anhand der Monitoring-Daten 2000 von Schröder et al. (2008) sowie für alle Teilnehmerstaaten des Moosmonitoring anhand der Messkampagne 2005 von Holy et al. (2009a). Diese Untersuchungen sowie die vorliegende Studie zeigen, dass die Verknüpfung der Messdaten mit den Eigenschaften der Moossammelorte sowie deren Umgebung plausible Erklärungen für die lokale und regionale Messwertvarianz liefern. Die Aussagekraft dieser Erklärungen ist gegenüber den bis zum Jahr 2008 veröffentlichten Auswertungen dadurch gestiegen, dass Informationen über die Emissionen verfügbar wurden und sich in die Auswertungen erstmals einbeziehen ließen (Holy et al. 2009a). Wenn dies auch mit den europaweiten Modellierungen der Deposition ermöglicht wird, darf erwartet werden, dass die Daten aus dem Moosmonitoring die Präzision der Depositionsmodellierung erhöhen (Ilyin 2009). Dies ist erforderlich, denn die intrinsische Unsicherheit der Modellierung der Gesamtdeposition mit den EMEP-Modellen beträgt für $\mathrm{Pb}$ und $\mathrm{Cd}$ rund $30 \%$, für $\mathrm{Hg}$ rund $40 \%$. Dies bedeutet für Deutschland, dass die Depositionsmodellierungen für das Bundesgebiet räumlich nicht ausreichend sind.

Die Entscheidungsbaumverfahren identifizieren einen ziemlich festen Kern mehrerer mit der Metall-Bioakkumulation assoziierter Faktoren, deren Relevanz räumlich etwas unterschiedlich sein kann. In Frankreich und Deutschland werden die höchsten MMI-Werte für Gegenden mit hohem Flächenanteil urbaner Landnutzung ermittelt. Während in Frankreich auch eine positive Korrelation der MMI (2005) mit der Höhenlage über NN gefunden wurde (Holy et al. 2009b), konnte dies in der vorliegenden Untersuchung anhand der Daten aus 2005 ebenso wenig bestätigt werden wie von Pesch et al. (2007b) auf Grundlage der Daten aus dem Jahr 2000. Die deutschen Befunde sind im Einklang mit Coşkun et al. (2005) und Schmidt-Grob et al. (1991). Gerdol et al. (2002) sowie Sucharova und Suchara (2004) berichten über negative Korrelationen zwischen Metallkonzentrationen in Moosen und der Höhenlage der beprobten Orte. Die französischen Befunde werden hingegen von Šoltès (1998), Zechmeister (1994, 1995), Zechmeister et al. (2003) sowie Gerdol and Bragazza (2006) gestützt.

Wie in dieser Untersuchung haben Analysen der deutschen Moosmonitoring-Daten der Jahre 1990, 1995 und 2000 belegt, dass die Moos-Arten mit den Metallgehalten korrelieren (Kleppin et al. 2008a; Schröder et al. 2008). Wie auch für Frankreich wurde offenbar, dass Pleuozium schreberi und Scleropodium purum geringere Metallgehalte aufweisen als Hypnum cupressiforme. Hierbei ist jedoch zu beachten, dass die miteinander verglichenen Moosarten nicht jeweils von denselben, sondern von unterschiedlichen Standorten stammen und die in ihnen gemessenen Stoffgehalte folglich nicht zwingend mit der Moosart, sondern mit den Depositionsverhältnissen und anderen akkumulationsrelevanten Merkmalen der unterschiedlichen Sammelorte zusammenhängen können. Leblond und Rausch de Traubenberg (2006, 
2007) weisen für an 15 Standorten gesammelte Proben von Pleuozium schreberi, Hypnum cupressiforme und Thuidium tamariscinum signifikant unterschiedliche Metallgehalte zwischen den Moosarten nach (Wilcoxon-Test, $\mathrm{p}<0.05$ ). Doch die Arten mit den höchsten Gehalten waren nicht bei allen Elementen dieselben, die Akkumulationseigenschaften sind also art- und stoffspezifisch. Artspezifische Unterschiede werden ferner von Castello (2007), Olajire (1998) sowie Zechmeister et al. (2003) berichtet. Rühling und Tyler (1968) sowie Ashmore et al. (2000) konnten dies nicht bestätigen.

Eine geringe Dichte der Moosdecke wurde in Frankreich und Deutschland als weiterer wichtiger Standortfaktor für die Metallakkumulation identifiziert (Holy et al. 2009b, Pesch et al. 2007b). Dies wird durch Castello (2007) und Steinnes (1995) bestätigt. Onianwa (2001) und Poikolainen (2004) weisen darauf hin, dass hier auch Wechselwirkungen zwischen hohen Einträgen und geringer Dichte der Moosdecke bestehen können.

\section{Schlussfolgerungen}

Die Daten des Moosmonitorings sind ein umweltpolitisch und praxisrelevanter Beitrag zum Schwermetall- und zum Multi-Komponenten-Protokoll der CLRTAP. Das Moosmonitoring belegt flächendeckend die Effekte der Luftreinhaltepolitik an den Wirkorten von Schadstoffen. Dies ist eine Daueraufgabe. Ihre Erfüllung gibt der Umweltpolitik die Möglichkeit, vorsorgend $\mathrm{zu}$ handeln und überraschenden Entwicklungen nachzugehen. Nur das Moosmonitoring registrierte den Anstieg einiger Metallanreicherungen in mehreren europäischen Staaten (u. a. in Deutschland) von 2000 nach 2005. In keinem anderen Messprogramm werden so verdichtet flächenbezogene Daten über ein breites, ökotoxikologisch relevantes Stoffspektrum erhoben. Die räumliche Auflösung von Umweltinformationen ist ein wesentliches Kriterium für ihre Nutzbarkeit im Vollzug umweltpolitischer Maßnahmen auf Bundes- und Länderebene.

Das Moosmonitoring erfasst anders als technische Oberflächen pflanzenverfügbare Inhaltsstoffe von Feinstaub. Physikalische Messnetze (z.B. Deposition, ICP Forests Level II, Umweltprobenbank) können nur an wenigen Orten Daten erheben, liefern gegenüber dem Moosmonitoring jedoch zeitlich besser aufgelöste Informationen. Die hohe räumliche Auflösung der Daten aus dem Moosmonitoring gegenüber derjenigen aus anderen Messnetzen ist für die räumliche Modellierung jedoch nützlich, um räumliche Lücken zu schließen. Für die Modellierung der Stoffeinträge in Ökosysteme durch Deposition liefert das Moosmonitoring derzeit die einzige Datengrundlage für eine Validierung der räumlichen Differenzierung auf kontinentaler, nationaler und regionaler Ebene.

Anders als Depositionsmessungen liefert das Moosmonitoring Informationen darüber, welche Stoffe in welcher Höhe am Wirkort/Schutzgut Pflanze (Moos) ankommen. Die Bioakkumulation von Stoffen in der Umwelt ist für die ökotoxikologische Bewertung der Erheblichkeit von Stoffeinträgen z. B. in Naturschutzgebieten und FFH-Gebieten aussagekräftiger als Depositionsdaten. Deshalb werden in den Bundesländern die Moosmonitoring-Daten den berechneten Critical Loads bei der Beurteilung der Erheblichkeit von Stoffeinträgen für Schutzgebiete vorgezogen. Das Moosmonitoring liefert nicht nur die Bestimmung der Umweltkonzentration von Stoffen, sondern immissionsschutzrechtlich relevante Beiträge zur Wirkungsermittlung. Die Stoffanreicherung in einem Organismus ist nach Bundes-Immisionsschutzgesetz (BImSchG) Teil einer potenziellen Wirkung. Insofern ist das passive Expositionsmonitoring mit Moosen insbesondere im Hinblick auf Vorsorge wichtig. Technische Depositionsmessungen und die Bestimmung der Akkumulation von Stoffen in Ökosystemkompartimenten sind komplementär zueinander: Sie liefern Daten zu verschiedenen, aufeinanderfolgenden Schritten in der Prozesskette Emission, Immission, Deposition sowie Akkumulation/Wirkung. Das standardisierte Biomonitoring von Luftverunreinigungen mit Bodenmoosen bildet ein wichtiges Bindeglied zwischen technischen Depositionsmessungen und biologischen Wirkungen. Insofern ist das Moosmonitoring für ein flächendeckendes Screening der stofflichen Exposition von umwelt- und naturschutzrechtlichen Schutzgütern besonders geeignet. Für Quecksilber und viele andere seltene Metalle, die zum Teil auch von humantoxikologischer Bedeutung sind, ist das Moosmonitoring derzeit die einzige flächenbezogene Informationsquelle zur räumlichen Struktur der Exposition, da in anderen Programmen nur wenige Schwermetalle und diese oft nur optional und an wenigen Orten gemessen werden. Die Daten des Moosmonitorings liegen europaweit vergleichbar und einheitlich vor.

Das Moosmonitoring ist im Vergleich zu Depositionsmessungen ein sehr kostengünstiges Verfahren zur Identifizierung von Hotspots und zeitlichen Trends der Schwermetall- und Stickstoffexposition. Die Bioakkumulation auch selten gemessener Schadstoffe mit humantoxikologischer Bedeutung (z. B. Hg, Sb, As, Al, V) kann leicht bestimmt werden. Für die engmaschige, flächendeckende Darstellung der Stoffexposition besitzt das Moosmonitoring ein Alleinstellungsmerkmal. Das Moosmonitoring liefert als einziges Messnetz deutschlandweite Daten über die Stickstoffexposition - auch in Schutzgebieten.

Ein deutschlandweiter Schwermetallindikator auf Grundlage der Moosmonitoring-Daten liegt für einzelne Naturräume und Schutzgebiete vor. Dies wurde mit den BergerhoffDaten bis heute nicht erreicht, denn diese Daten werden bis heute unverknüpft von den Bundesländern verwaltet, und bundesweit harmonisierte und zusammengeführte Ergebnisse sind nicht zugänglich und gestatten keine praxisrelevante räumliche Differenzierung. Zudem treten bei der Verwendung der Moosmonitoring-Daten im Gegensatz zu 
dem vom LIKI favorisierten Bergerhoff-Messnetz oder zu anderen methodisch sehr unterschiedlich angelegten - daher eigentlich nicht deutschlandweit integrierbaren - Depositionsmessnetzen keine Verzerrungen durch flächendisproportionale Messstandort-Verteilungen auf. Naturräumliche Differenzierungen, die für die Bewertung der Stoffeinträge essenziell sind, werden also durch das Moosmonitoring, nicht jedoch durch das Bergerhoff-Messnetz ermöglicht.

\section{Empfehlungen und Ausblick}

Das ICP Vegetation regt an, das Moosmonitoring zukünftig enger mit dem Human-Biomonitoring (in Deutschland z. B. Umweltprobenbank/Teilbank Humanprobenund Kinder-Umwelt-Survey) zu verknüpfen (Zusammenarbeit mit ICP Task Force on Health). Die räumlich differenzierte Erfassung der Beziehungen zwischen der inneren Exposition von Probanden durch Human-Biomonitoring einerseits und der äußeren Exposition in Gebäuden (Indoor-Exposition) und im Freien (Outdoor-Exposition) durch Umweltmonitoring andererseits fehlt sowohl im Umwelt-Gesundheitssurvey als auch bei den Messungen der Umweltprobenbank/Teilbank Humanproben bislang weitgehend. Eine methodische Verknüpfung von Indoor- und Outdoor-Expositionsmonitoring könnte durch die Verwendung derselben Schadstoffrezeptoren im Indoor- und im Outdoor-Bereich ermöglicht werden. Untersuchungen zeigen, dass Moos ein sehr gut geeignetes Rezeptormedium für das Indoor- und Outdoor-Expositionsmonitoring ist. Ziel sollte es sein, eine Biomonitoring-Methode für die Langzeiterfassung organischer und anorganischer Schadstoffe mit einem Rezeptor zu entwickeln und zu erproben, der über ein breites Spektrum von Stoffen und Klimabedingungen im Indoor- und Outdoor-Monitoring einsetzbar ist und die Beziehungen zwischen innerer Exposition und äußerer Exposition (Indoor, Outdoor) ermöglicht (Zechmeister et al. 2007).

\section{Literatur}

Ashmore M, Bell S, Fowler D, Hill M, Jordan C, Nemitz E, Parry S, Pugh B, Reynolds B, Williams J (2000) Survey of the UK metal content of mosses 2000. Part II of EPG 1/3/144 final contract report. Development of a critical load methodology for toxic metals in soils and surface waters: stage II. University of Bradford, UK

Auerswald K, Wittmer MHOM, Männel TT, Bai YF, Schäufele R, Schnyder H (2009) Large regional scale variation in C3/C4 distribution pattern of Inner Mongolia steppe is revealed by grazer wool carbon isotope composition. Biogeosci Discuss 6:545-574

Baltes-Götz B (2004) Entscheidungsbaumanlyse mit AnswerTree 3.1. http://www.uni-trier.de/urt/user/baltes/docs/at/v31/at31.pdf.

Bealey W, Cape JN, Leith ID, Long S, Kinnerlsey RP (2008a) Air quality outcomes in pollution regulation: strengths, limitations and potential. Science Report SC030175/SR1, CEH Project Number: C02600. Environment Agency, Bristol 1-47

Bealey WJ, Long S, Spurgeon DJ, Leith I, Cape JN (2008b) Review and implementation study of biomonitoring for assessment of air quality outcomes. Science Report - SC030175/SR2. Environment Agency, Bristol 1-170

Breimann L, Friedmann JA, Olshen RA, Stone CJ (1984) Classification and regression trees. Belmont, CA: Wadsworth

Cambardella CA, Moorman TB, Novak JM, Parkin TB, Karlan DL, Turco RF, Konopka AE (1994) Field-scale variability of soil properties in central Iowa soils. Soil Sci Soc America J 58:1501-1511

Castello M (2007) A comparison between two moss species used as transplants for airborne trace element biomonitoring in NE Italy. Environ Monit Assess 133:267-276

Coşkun M, Frontasyeva MV, Steinnes E, Cotuk AY, Pavlov SS, Coșkun M, Sazonov AS, Cayir A, Belivermis M (2005) Atmospheric Deposition of Heavy Metals in Thrace Studied by Analysis of Moss (Hypnum cupressiforme). B Environ Contam Tox 74:201-209

Diehl M S, Beard K (2009) Spatial analysis of atmiospheric deposition and terrestrial accumulation of mercury within Acadia National Park. Spatial Information Sciences Engineering, University of Maine, Orono. Northeastern Section - 44th Annual Meeting (2224 March 2009), Session No. 48: GIS Applications in Geoscience Teaching, Research and Map Production (http://gsa.confex.com/ gsa/2009NE/finalprogram/abstract_155805.htm)

Forster E-M, Matthies M, Brüggemann R (1993) Optimierung eines Bioindikator-Meßnetzes mit geostatistischen Methoden und einem geographischen Informationssystem. Umweltwiss Schadst Forsch 5:286-294

Fränzle O, Schimming CG (2008) Element fluxes in atmosphere, vegetation and soil. In: Fränzle O, Kappen L, Blume H P, Dierssen $\mathrm{K}$ (Eds) Ecosystem organiszation of a complex landscape. Long-term research in the Bornhöved Lake District, Germany. Berlin, Springer 169-205

Fränzle O, Straškraba M, Jørgensen S E (1995) Ecology and ecotoxicology Ullmann's encyclopedia of industrial chemistry Vol B7, Weinheim, VCH 19-154

Fränzle S (2007) Prinzipien und Mechanismen der Verteilung und Essentialität von chemischen Elementen in pflanzlicher Biomasse - Ableitungen aus dem Biologischen System der Elemente. Habil-Schr Lehrstuhl für Landschaftsökologie, Hochschule Vechta

Funk W, Dammann V, Donnevert G (2006) Quality assurance in analytical chemistry. Applications in environmental, food and materials analysis, biotechnology and medical engineering. 2nd ed, Weinheim, Wiley-VCH

Genßler L, Rademacher J, Rammert U (2001) Arbeitskreis der Landesanstalten und -ämter Konzeption der künftigen Aufgabenbereiche. Umweltwiss Schadst Forsch 13:1-4

Gerdol R, Bragazza L (2006) Effects of altitude on element accumulation in alpine moss. Chemosphere 64:810-816

Gerdol R, Bragazza L, Marchesini R (2002) Element concentrations in the forest moss Hylocomium splendens: variation associated with altitude, net primary production and soil chemistry. Environ Pollut 116:129-135

Guderian R, Gunkel G (Hrsg. 2001) Handbuch der Umweltveränderungen und Ökotoxikologie: Band 2b. Springer, Berlin, $563 \mathrm{~S}$

Hagl S (2008) Schnelleinstieg Statistik - Daten erheben, analysieren, präsentieren. Freiburg, Berlin, München

Harmens H (2005) Monitoring of atmospheric deposition in Europe using bryophytes. Monitoring manual 2005/2006 survey. Bangor, UK

Harmens H, Norris D and the participants of the moss survey (2008) Spatial and temporal trends in heavy metal accumulation in mosses in Europe (1990-2005). Programme Coordination Centre for the ICP Vegetation, Centre for Ecology and Hydrology, Environment Centre Wales, Bangor, UK

Herpin U (1997) Moose als Bioindikatoren von Schwermetalleinträgen. Möglichkeiten und Grenzen für flächendeckende und zeitabhängige Aussagen. Dissertation, Universität Osnabrück 
Herpin U, Siewers U, Markert B, Rosolen V, Breulmann G, Bernoux M (2004) Second German heavy-metal survey by means of mosses, and comparison of the first and second approach in Germany and other European countries. Env Sc Pollut Res 11:57-66

Holy M, Pesch R, Schröder W, Harmens H, Alber R, Aleksiayenak Y, Blum O, Coskun M, Dam M, de Temmermann L, Frolova M, Frontasyeva M, Grodzińska K, Jeran Z, Kubin E, Kvietkus K, Leblond S, Liiv S, Magnússon S, Maňkovská B, Miodrag K, Rühling Å, Santamaria J, Steinnes E, Suchara I, Szymon K, Thöni L, Urumov V, Yurukova L, Zechmeister (2009a) Europe-wide analysis of factors influencing the spatial variation of cadmium, lead and mercury concentrations in mosses In: Harmens H, Mills G, Menichino N, Bender J, Weigel H (Eds) 22nd Task Force Meeting of the ICP Vegetation, 2-5 February 2009, Braunschweig. ICP Vegetation Programme Coordination Centre, Centre for Ecology and Hydrology, Bangor, UK 26

Holy M, Leblond S, Pesch R, Schröder W (2009b) Assessing spatial patterns of metal bioaccumulation in France by means of an exposure index. Environ Sci Poll Res (accepted)

ICP Vegetation (2005) Heavy metals in European mosses: 2005/2006 survey. Monitoring manual. ICP Vegetation Coordination Centre, Centre for Ecology and Hydrology. Bangor, UK

Ilyin I (2009) Modelled EMEP heavy metal deposition vs moss measurements: Evaluation of spatial patterns and log-term temporal trends In: Harmens H, Mills G, Menichino N, Bender J, Weigel H (Eds) 22nd Task Force Meeting of the ICP Vegetation, 2-5 February 2009, Braunschweig. ICP Vegetation Programme Coordination Centre, Centre for Ecology and Hydrology, Bangor, UK 27

Johnston K, Ver Hoef JM, Krivoruchko K, Lucas N (2001) Using ArcGIS Geostatistical Analyst. Redlands

Kass GV (1980) An exploratory technique for investigating large quantities of categorial data. Appl Stat 29:199-127

Keil M, Kiefl R, Strunz G (2005) CORINE Land Cover 2000 - Germany. Final Report. German Aerospace Center, German Remote Sensing Data Center, Oberpfaffenhofen

Kerry R, Oliver M A (2008) Determinig nugget:sill ratios of standardized variograms from aerial photographs to krige sparse soil data. Precision Agric 9:33-56

Kleppin L, Pesch R, Schröder W (2008a) CHAID-Models on boundary conditions of metal accumulation in mosses collected in Germany 1990, 1995 and 2000. Atmos Environ 42:5220-5231

Kleppin L, Schröder W, Pesch R, Schmidt G (2008b) Entwicklung und Erprobung einer Metadaten- und WebGIS-Applikation für das Expositionsmonitoring mit Moosen in Deutschland Ein Beitrag zum LTER-Netzwerk. Umweltwiss Schadst Forsch 20:38-48

Kostka-Rick R, Leffler U S, Markert B, Herpin U, Lusche M, Lehrke J (2001) Biomonitoring zur wirkungsbezogenen Ermittlung der Schadstoffbelastung in terrestrischen Ökosystemen Konzeption, Durchführung und Beurteilungsmaßstäbe im Rahmen von Genehmigungsverfahren. Umweltwiss Schadst Forsch $12: 5-12$

Le Corre V, Roussel G, Zanetto A, Kremer A (1998) Geographical structure of gene diversity in Quercus petrea (Matt) Liebl III Patters of variation identified by geostatistical analyses. Hederdity $80: 464-473$

Leblond S, Rausch de Traubenberg C (2006) Etude des retombées atmosphériques de métaux en France. Estimation par dosage dans des mousses. Campagne $2006 \mathrm{du}$ dispositif BRAMM (Biosurveillance des Retombées Atmosphériques Métalliques par les Mousses), Marché ADEME N 0562C0036, 1er Rapport Intermédiaire, Paris

Leblond S, Rausch de Traubenberg C (2007) Etude des retombées atmosphériques de métaux en France. Estimation par dosage dans des mousses. Campagne $2006 \mathrm{du}$ dispositif BRAMM (Biosurveillance des Retombées Atmosphériques Métalliques par les
Mousses) - Paris. (Marché ADEME N 0562C0036, 2ième Rapport Intermédiaire)

Lindberg SE, Turner RR (1988) Factors influencing atmospheric deposition, stream export, and landscape accumulation of trace metals in forested watersheds. Water Air Soil Pollut 39:123-156

MARSEP (International Manure and Refuse Sample Exchange Programme) (2000) Quarterly report 20003 Department Environmetal Sciences, Sub-Department of Soil Science and Plant Nutrition, Agricultural University Wageningen

Matheron G (1965) Les variables régionalisées et leur estimation. Paris: Masson

Mohr K (1999) Passives Monitoring von Stickstoffeinträgen in Kiefernforsten mit dem Rotstengelmoos (Pleurozium schreberi (Brid) Mitt). Umweltwiss Schadst Forsch 11:267-274

Mohr K (2007) Biomonitoring von Stickstoffimmissionen. Möglichkeiten und Grenzen von Bioindikationsverfahren. Umweltwiss Schadst Forsch 19(4):255-264

Olajire A (1998) A survey of heavy metal deposition in Nigeria using the moss monitoring method. Environ Int 24(8):951-958

Onianwa PC (2001) Monitoring atmospheric metal pollution: a review of the use of mosses as indicators. Environ Monit Assess $71: 13-50$

Osborn D, Weeks J M, Hankard P, Dale L (2000) Potential uses of biomonitoring in pollution control - An introductory guide. Environment Agency Technical Report 1-319

Pesch R (2003) Geostatistische und multivariat-statistische Analyse des Moos-Monitoring 1990, 1995 und 2000 zur Ableitung von Indikatoren für die Bioakkumulation atmosphärischer Metalleinträge in Deutschland. Dissertation, Hochschule Vechta

Pesch R, Holy M, Schröder W (2008) Statistical investigation of the atmospheric accumulation of antimony and nitrogen in mosses In: Car A, Griesebner G, Strobl J (Eds): Geospatial Crossroads @ GI_Forum ,08: Proceedings of the Geoinformatics Forum Salzburg Heidelberg, Wichmann 227-236

Pesch R, Schmidt G, Schröder W, Aden C, Kleppin L, Holy M (2007a) Development, implementation and application of the WebGIS MossMet. In: Tochtermann K, Scharl A (Eds) The geospatial web. How geo-browsers, social software and the Web 2.0 are shaping the network society. London, Springer, 191-200

Pesch R, Schröder W (2006) Integrative exposure assessment through classification and regression trees on bioaccumulation of metals, related sampling site characteristics and ecoregions. Ecol Inf $55-65$

Pesch R, Schröder W, Dieffenbach-Fries H, Genßler L, Kleppin L (2008) Optimierung des Moosmonitoring-Messnetzes in Deutschland Umweltwiss Schadst Forsch 20:49-61

Pesch R, Schröder W, Genßler L, Göritz A, Holy M, Kleppin L, Matter Y (2007b) Moos-Monitoring 2005/2006: Schwermetalle IV und Gesamtstickstoff. - Berlin (Umweltforschungsplan des Bundesministers für Umwelt, Naturschutz und Reaktorsicherheit. FuE-Vorhaben 20564 200, Abschlussbericht, im Auftrag des Umweltbundesamtes); 90 S., 11 Tab., 2 Abb. (Texteil); 51 S. + 41 Karten, 34 Tabellen, 46 Diagramme (Anhangsteil)

Poikolainen J (2004) Mosses, epiphytic lichens and tree bark as biomonitors for air pollutants - specifically for heavy metals in regional surveys. Dissertation, University of Oulu

Rühling A, Tyler G (1968) An ecological approach to the lead problem. Botaniska Notiser 121:321-343

Rühling A, Tyler G (1969) Ecology of heavy metals - a regional and historical study. Botaniska Notiser 121:248-259

Rühling A, Tyler G (1970) Sorption and retention of heavy metals in the woodland moss Hylocomium splendens (Hedw.) Br Et Sch Oikos 21:248-342

Schmidt-Grob I, Thöni L, Hertz J (1991) Übersicht über die Deposition von Arsen, Blei, Cadmium, Chrom, Cobalt, Eisen, Kupfer, Molybdän, Nickel, Quecksilber, Schwefel, Thallium, Vanadium 
und Zink in der Schweiz mit Hilfe von Moosen als Biomonitoren. Forschungsstelle für Umweltbeobachtung, Birmensdorf, Switzerland

Schröder W, Anhelm P, Bau H, Bröcker F, Matter Y, Mitze R, Mohr K, Peichl L, Peiter A, Peronne T, Pesch R, Roostai AH, Roostai Z, Schmidt G, Siewers U (2002): Untersuchung von Schadstoffeinträgen anhand von Bioindikatoren. Aus- und Bewertung der Ergebnisse aus dem Moos-Monitoring 1990, 1995 und 2000. Umweltforschungsplan des Bundesministers für Umwelt, Naturschutz und Reaktorsicherheit. FuE-Vorhaben 20064 218, Abschlussbericht Band 1 bis 3 + Synthesebericht, im Auftrag des Umweltbundesamtes. Berlin, 221 S, 29 Tab, 94 Abb.

Schröder W, Englert C, Pesch R, Zechmeister HG, Thöni L, Suchara I, Maňkovská B, Jeran Z, Harmens H, Grodzinska K, Alber R (2008) Metallakkumulation in Moosen: Standörtliche und regionale Randbedingungen des Biomonitoring von Luftverunreinigungen. Umweltwiss Schadst Forsch 20:120-132

Schröder W, Garbe-Schönberg CD, Fränzle O (1991) Die Validität von Umweltdaten Kriterien für ihre Zuverlässigkeit: Repräsentativität, Qualitätssicherung und -kontrolle. Umweltwiss Schadst Forsch 3:237-241

Schröder W, Hornsmann I, Pesch R, Schmidt G, Fränzle S, Wünschmann S, Heidenreich H, Markert B (2008) Moosmonitoring als Spiegel der Landnutzung? Stickstoff- und Metallakkumulation in Moosen zweier Regionen Mitteleuropas. Umweltwiss SchadstForsch 20:62-74

Schröder W, Pesch R (2004) The 1990, 1995 and 2000 moss monitoring data in Germany and other European countries Trends and statistical aggregation of metal accumulation indicators. Gate Environ Health Sci, June 2004:1-25, DOI: http://dxdoiorg/101065/ ehs200406011

Schröder W, Pesch R (2005) Time series of metals in mosses and their correlation with selected sampling site-specific and ecoregional characteristics in Germany. Env Sci Pollut Res 12:159-167

Schröder W, Pesch R, Schmidt G (2004) Soil monitoring in Germany Spatial representativity and methodical comparability. J Soils Sediments 4:49-58

Schröder W, Schmidt G (2000) Raumgliederung für die Ökologische Umweltbeobachtung des Bundes und der Länder. Umweltwiss Schadst Forsch 12:237-243

Siewers U, Herpin U, Straßburger S (2000) Schwermetalleinträge in Deutschland Moos-Monitoring 1995 Teil 2. Geologisches Jahrbuch, Sonderhefte, Heft SD 3. Stuttgart, Bornträger

Smodiš B, Bleise A (2007) IAEA quality control study on determining trace elements in biological matrices for air pollution research. J Radioan Nucl Ch 271:269-274

Šoltès R (1998) Correlation between altitude and heavy metal deposition in the Tatra Mountains (Slovakia). Biologia 53:85-90
Sparks T (2000) Statistics in ecotoxicology. Ecological and environmental toxicology series. Weinheim, Wiley-VCH

Steinnes E (1995) A critical evaluation of the use of naturally growing moss to monitor the deposition of atmospheric metals. Sci Total Environ 160/161:243-249

Steinnes E, Rühling Å, Lippo H, Mäkinen A (1997) Reference materials for large-scale metal deposition surveys. Accredit Qual Assur 2(5):243-249

Stevens S S (1946) On the theory of scales of measurement. Science 103:677-680

Sucharova J, Suchara I (2004) Bio-monitoring the atmospheric deposition of elements and their compounds using moss analysis in the Czech Republic. Results of the international bio-monitoring programme UNECE ICP-Vegetation 2000. Part I: Elements required for the bio-monitoring programme. Acta Pruhoniciana 77, $135 \mathrm{~S}$

Tyler G. (1990) Bryophytes and heavy metals: a literature review. Bot J Linnean Soc 104:231-253

van Dijk D, Houba VJG (1999) Wageningen evaluating programmes for analytical laboratories (WEPAL) Arh hig rada toksikol 50:31-36

Wappelhorst O, Korhammer S, Leffler U S, Markert B (2000) Ein Moosbiomonitoring zur Ermittlung atmosphärischer Elementeinträge in die Euroregion Neiße (D, PL, CZ) UWSF - Z Umweltchem Ökotox 12(4):191-200

Webster R, Oliver MA (2001) Geostatistics for environmental scientists. Chichester, New York, Weinheim, Brisbane, Singapore, Toronto, Wiley

Zechmeister HG (1994) Biomonitoring der Schwermetalldepositionen mittels Moosen in Österreich. Monographien des Umweltbundesamtes $42: 1-168$

Zechmeister HG (1995) Correlation between altitude and heavy metal deposition in the Alps. Environ Pollut 89:73-80

Zechmeister HG, Dullinger S, Hohenwallner D, Riss A, HanusIllnar A, Scharf S (2006) Pilot study on road traffic emissions (PAHs, heavy metals) measured by using mosses in a tunnel experiment in Vienna, Austria. Env Sc Pollut Res 13:398-405

Zechmeister HG, Grodzinska K, Szarek-Lukaszewska G (2003) Bryophytes. In B.A. Markert, A. M. Breure, H. G. Zechmeister (Eds.), Bioindicators \& biomonitors. Principles, concepts and applications. Amsterdam, Elsevier 329-375

Zechmeister HG, Köllensperger G, Hann S, Hanus-Illnar A, Scharf S, Schneider J, Neuberger M, Moshammer H, Hohenwallner D, Schierl R, Schröder W, Schmidt G, Pirintsos SA, Loppi S, Gaggi C, Nante N, Namiesnik J, Zygmunt B, Jeran Z, Perharic L, Künzli N, Frontasyeva M, Lyapunov S, Ilchenko I, Harmens H (2007) INDOMO. New tool for monitoring indoor pollutants with health relevance across Europe. Wien

Zhao B, Cai Q (2004) Geostatistical analysis of chlorophyll a in freshwater ecosystems. J Freshwater Ecol 19:613-621 